\title{
On The Throughput-Reliability Tradeoff for Amplify-and-Forward Cooperative Systems
}

\author{
Jun Li, Member, IEEE, Wen Chen, Senior Member, IEEE, Aria Nosratinia, Fellow, IEEE, \\ and Jinhong Yuan, Senior Member, IEEE
}

\begin{abstract}
This paper investigates the throughput-reliability tradeoff (TRT) for dual-hop amplify-and-forward relay systems with one source, one destination, and multiple relays, and its relationship with the diversity-multiplexing tradeoff (DMT). The TRT was proposed in the context of MIMO block fading channels to reveal the interplay between the signal-to-noise ratio (SNR), rate $R$, and outage probability that are not accessible through the DMT. The contributions of this paper include the calculation of the TRT expressions for two classes of amplifyand-forward protocols: the slotted amplify-and-forward and the non-orthogonal amplify-and-forward. Based on the derived expressions, relationships between the SNR, rate and outage probability are explored. The relationship between the TRT and the DMT is investigated. One of the goals of the TRT is to predict the slope and offset of the outage vs. SNR set of curves parameterized by different rates. We verify the accuracy of the TRT predictions in the context of amplify-and-forward relays.
\end{abstract}

Index Terms-Cooperative systems, amplify-and-forward, diversity-multiplexing tradeoff, throughput-reliability tradeoff.

\section{INTRODUCTION}

$\mathbf{M}$ IMO techniques were known to provide gains in terms of rate (spatial multiplexing) as well as reliability and diversity (space-time codes). Zheng and Tse [1] took a significant step in bridging these two areas of investigation by the introduction of the concept of diversity-multiplexing tradeoff (DMT). Among other insights, this work showed that it is possible to mix and match the two types of gains in MIMO systems (rate and reliability) in a manner prescribed by the DMT. The DMT assumes a family of codes, in which diversity gain $d$ and multiplexing gain $r$ are defined by

$$
d \triangleq-\lim _{\rho \rightarrow \infty} \frac{\log \left(P_{e}(\rho)\right)}{\log \rho} \quad \text { and } \quad r \triangleq \lim _{\rho \rightarrow \infty} \frac{R(\rho)}{\log \rho},
$$

Manuscript received April 24, 2012; revised September 9 and October 22, 2012. The associate editor coordinating the review of this paper and approving it for publication was A. Nallanathan.

$\mathrm{J}$. Li is with the School of Electrical and Information Engineering, The University of Sydney, NSW, 2006, Australia (e-mail: jun.li1@ sydney.edu.au).

W. Chen is with the Department of Electronic Engineering, Shanghai Jiao Tong University, Shanghai, 200240, China (e-mail: wenchen@sjtu.edu.cn).

A. Nosratinia is with the Department of Electrical Engineering, The University of Texas at Dallas, Richardson, TX, 75083, USA (e-mail: aria@utdallas.edu).

J. Yuan is with the School of Electrical Engineering and Telecommunications, The University of New South Wales, Sydney, NSW 2052, Australia (e-mail: j.yuan@unsw.edu.au).

The work in this paper is partially supported by the National 973 Projects 2012CB316106 and 2009CB824904, by NSF China 60972031 and 61161130529, and by the Australian Research Council (ARC) Discovery Project DP110104995.

Digital Object Identifier 10.1109/TCOMM.2013.012913.120285 where $\rho, P_{e}(\rho)$ and $R(\rho)$ represent the signal-to-noise ratio (SNR), codeword error probability, and transmission rate, respectively (In this paper log is base 2). A scheme's DMT means that at the multiplexing gain $r$, the diversity gain is equivalent to $d(r)$. In [1], the diversity multiplexing tradeoff was developed for MIMO systems subject to a lower bound on the codeword length, resulting in a piecewise-linear characteristic in the diversity-multiplexing plane.

Relay systems, which are sometimes roughly represented as a method of achieving "distributed MIMO gains," may also benefit from DMT analysis. A few instances of the DMT of one-antenna relay systems were presented by the early work of Laneman et. al [2], and the DMT analysis of multi-antenna relays was analyzed by Yuksel and Erkip [3]. The DMT of various relaying scenarios has received much attention, among others in [4]-[13]. Often times the DMT analysis is simplified by allowing the use of outage probability $P_{o}$ instead of error probability $P_{e}$ under the condition of long codewords, and the same approach is taken in this paper.

Although DMT is a powerful framework, there are questions that it leaves unanswered. For instance, Azarian and El Gamal [14], [15] raised the natural question: how much rate (or reliability) is gained, asymptotically, by a $3 \mathrm{~dB}$ increase in SNR in MIMO systems? In general DMT does not answer these two questions directly, instead, it tells us that at operating point $(r, d(r))$ we get a combination of $r$ additional bit per channel use (bpcu) and $2^{-d(r)}$ improvement in error. The reason for this is that the DMT framework only allows the rate of the (hypothetical) codebooks to increase linearly with $\log \rho$, thus limiting the type of questions that may be asked about the performance of the system. Instead, Azarian and El Gamal produced a framework where the codebook rates evolve more generally, in fact potentially

$$
\limsup _{\rho \rightarrow \infty} \frac{R}{\log \rho} \neq \liminf _{\rho \rightarrow \infty} \frac{R}{\log \rho} .
$$

The more general relationship between the three quantities $\{R$, $\left.\log \rho, P_{o}(R, \rho)\right\}$ in MIMO systems was called the throughputreliability tradeoff (TRT) [14]. The TRT was shown to have an operational meaning: the TRT reliably predicts the local slopes as well as the vertical displacement between error waterfall curves for MIMO systems calculated at various rates.

However, a very useful result as it is, TRT was only developed very specifically in the context of the MIMO channel, and there was no indication that it can be extended to other systems under fading. In [15], the authors posed the following open problem: "For MIMO channels, we established 


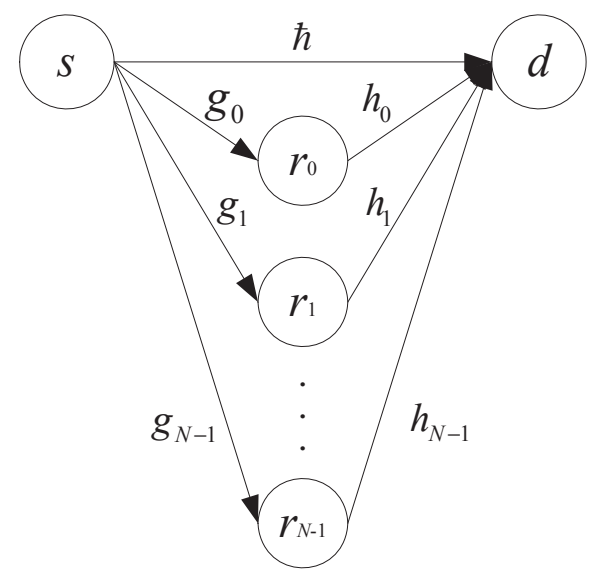

Fig. 1. Cooperative channels with $N$ isolated relays. All the channels are i.i.d Rayleigh distributed. There is no communications between the relays.

the correspondence between DMT and TRT formulations. It remains to see if such a correspondence exists for general channels or not." This open question motivates the present study on the throughput-reliability tradeoff for more general relay channels in cooperative systems.

In this paper, we study the TRT of the AF protocols in a relaying system. We will focus on two AF protocols, i.e., sequential slotted AF (SAF) protocol [16], [17] and nonorthogonal AF (NAF) protocol [4], [18]. We select the SAF because it is optimal in terms of DMT, and is more comprehensive than other AF protocols of this kind, e.g., in the SAF, signals from the source and the relay are always interfered at the destination. We use the NAF as an example to show that our TRT analysis in the SAF can be generalized to other AF protocols. We select the NAF because it is a widely studied AF protocol. Our main contribution in this paper is to investigate the TRT of the two AF protocols and find the connection between their TRT and DMT expressions. Specifically, for different relationship between the frame length $l$ and the number of the relays $N$ (e.g., $l \geq N$ and $l<N$ ), and for different relay selection schemes, we derive their TRT correspondingly. We compare the simulation results with the theoretic outage probability curves predicted by TRT. It is shown that, TRT accurately reveals the interplay between the triplets $R, \log \rho, P_{o}(R, \rho)$.

Notations: $(x)^{+}$represents $\max \{0, x\}, \mathbb{R}^{N}$ represents the set of real $N$-tuples, and $\mathbb{R}^{N+}$ denotes the set of non-negative $N$-tuples. For $\mathcal{O} \subseteq \mathbb{R}^{N}$, we denote the complementary set of $\mathcal{O}$ as $\mathcal{O}^{c}$, while $\mathcal{O} \cap \mathbb{R}^{N+}$ as $\mathcal{O}^{+}$. For two non-negative numbers $l$ and $N, l_{N}$ means $l \bmod N$. All the vectors are denoted by bold small letters and all the matrices are denoted by bold capital letters. $\mathbf{I}_{l}$ denotes the $l \times l$ identity matrix. $\mathbf{M}^{T}$ represents the transpose and $\mathbf{M}^{\dagger}$ represents the conjugate transpose of $\mathbf{M}$.

\section{System Model AND PREliminaries}

Fig. 1 shows a relaying system, which is composed of one source, one destination and $N(N>1)$ half-duplexing relays. We call the system as $1-N-1$ relaying network. We assume that each frame $\left[x_{0}, \cdots, x_{l-1}\right]^{T}$ transmitted by the source has $l$ symbols. The received signals at the destination during

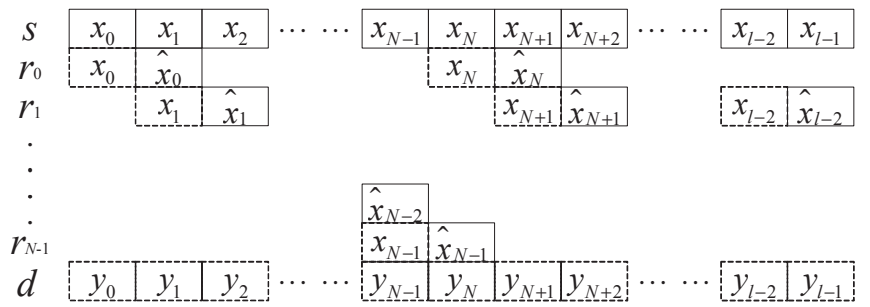

Fig. 2. Frame structure and scheduling strategy in the SAF protocol when $l>N$. The symbols inside of dashed-line boxes represent the received symbols and the symbols inside of the solid-line boxes represent the transmitted symbols.

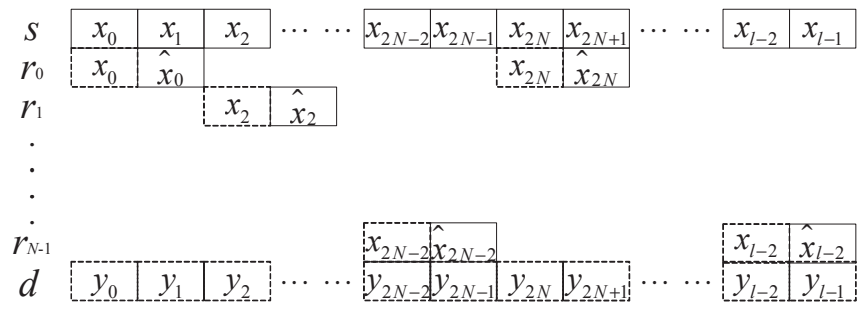

Fig. 3. Frame structure and scheduling strategy in the NAF protocol when $l>N$. The symbols inside of dashed-line boxes represent the received symbols and the symbols inside of the solid-line boxes represent the transmitted symbols.

a frame period are denoted as $\left[y_{0}, \cdots, y_{l-1}\right]^{T}$. The average symbol power at both the source and the relays is $P$. The Gaussian noises observed by each relay and the destination are i.i.d with the variances $\sigma_{w}^{2}$ and $\sigma_{v}^{2}$, respectively. The system SNR is defined as $\rho=\frac{P}{\sigma_{v}^{2}}$. All the channels are assumed to be Rayleigh $i . i . d$ with the variance one. The channel coefficients are constant during one frame period, but are independent from frame to frame. We use $g_{i}, \hbar$ and $h_{i}$ to denote the channels between the source and the $i$-th relay, the channel between the source and the destination, and the channels between the $i$-th relay and the destination.

We consider two AF protocols, i.e., the SAF protocol [16] and the NAF protocol [18]. In the SAF protocol, we consider a scheduling strategy shown in Fig. 2. In this strategy, the relays are scheduled in a round-robin manner and each relay is arranged to forward one symbol (or one time slot) in a round. We follow the isolated-relay assumption in [16] that the inter-relay interference is neglected. This is made possible when the relays are located and ordered in such a way that consecutive relays are separated as far as possible to minimize the inter-relay interference, and thus approximate the relayisolation condition.

For the $1-N-1$ relaying network with the NAF protocol, the scheduling strategy is shown in Fig. 3. We can see that the relays forward half the symbols in a frame, i.e., the relays receive and forward the symbols with even numbers of subscript in a frame. The relays are arranged in the roundrobin style and each relay is supposed to transmit one symbol in each round.

We start with the following definition and lemma.

Definition 1: Suppose SNR $\rho$ goes to infinity, we define 


$$
\begin{aligned}
& \boldsymbol{\Xi}=\left[\begin{array}{ccccc}
h_{0} b_{0} & \cdots & 0 & \cdots & 0 \\
\vdots & \ddots & \vdots & & \vdots \\
0 & \cdots & h_{j_{N}} b_{j_{N}} & \cdots & 0 \\
\vdots & & \vdots & \ddots & \vdots \\
0 & \cdots & 0 & \cdots & h_{(l-2)_{N}} b_{(l-2)_{N}}
\end{array}\right] \\
& \mathbf{H}=\left[\begin{array}{cccccccc}
\hbar & 0 & \cdots & 0 & 0 & \cdots & 0 & 0 \\
g_{0} h_{0} b_{0} & \hbar & \cdots & 0 & 0 & \cdots & 0 & 0 \\
\vdots & \vdots & & \vdots & \vdots & & \vdots & \vdots \\
0 & 0 & \cdots & \hbar & 0 & \cdots & 0 & 0 \\
0 & 0 & \cdots & g_{j_{N}} h_{j_{N}} b_{j_{N}} & \hbar & \cdots & 0 & 0 \\
\vdots & \vdots & & \vdots & \vdots & \vdots & \vdots & \vdots \\
0 & 0 & \cdots & 0 & 0 & \cdots & \hbar & 0 \\
0 & 0 & \cdots & 0 & 0 & \cdots & g_{(l-2)_{N}} h_{(l-2)_{N}} b_{(l-2)_{N}} & \hbar
\end{array}\right]
\end{aligned}
$$

$u(\rho) \doteq v(\rho)$ when

$$
\lim _{\rho \rightarrow \infty} \frac{\log (u(\rho))}{\log \rho}=\lim _{\rho \rightarrow \infty} \frac{\log (v(\rho))}{\log \rho} .
$$

Lemma 1: Suppose $\mu_{i}=\frac{\log \left(\rho\left|g_{i}\right|^{2}\right)}{R}, v_{i}=$ $\frac{\log \left(\rho\left|h_{i}\right|^{2}\right)}{R}$, and $\omega_{i}=\frac{\log \left(\rho\left|g_{i} h_{i}\right|^{2}\right)}{R}$, where $g_{i}$ and $h_{i}$ are Rayleigh i.i.d. Then the probability density function (PDF) of $\omega_{i}$, i.e., $p\left(\omega_{i}\right)$ can be written as

$$
p\left(\omega_{i}\right)=\frac{R \ln 2}{\rho} \cdot 2^{\left(\mu_{i}+v_{i}-\frac{\log \rho}{R}\right) R} \exp \left\{-\frac{2^{\mu_{i} R}+2^{v_{i} R}}{\rho}\right\} .
$$

Proof: Please refer to Appendix A.

\section{TRT THEOREMS FOR THE SAF PROTOCOL}

During a frame period, the received signals at the destination can be written as

$$
\mathbf{y}=\mathbf{H} \mathbf{x}+\left[\begin{array}{cc}
0 & \mathbf{o}_{l-1}^{T} \\
\mathbf{o}_{l-1} & \Xi
\end{array}\right] \mathbf{w}+\mathbf{v}
$$

where $\mathbf{y}=\left[y_{0}, \cdots, y_{l-1}\right]^{T}$ represents the length- $l$ vector of the received signals at the destination, $\mathbf{x}=\left[x_{0}, \cdots, x_{l-1}\right]^{T}$ represents the length- $l$ vector of source symbols, $\mathbf{w}$ represents the length- $l$ vector of noise samples observed by the relays, $\mathbf{o}_{(l-1)}$ represents the length- $(l-1)$ all zero vector, and $\mathbf{v}$ represents the length- $l$ vector of noise samples observed by the destination. The $(l-1) \times(l-1)$ matrix $\boldsymbol{\Xi}$ and the $l \times l$ matrix $\mathbf{H}$ are given by (3) and (4), respectively, where $0 \leq j<l-1$, the subscript $j_{N}$ means $j \bmod N$ (i.e., $g_{j_{N}}=$ $g_{(j \bmod N)}, h_{j_{N}}=h_{(j \bmod N)}$, and $\left.b_{j_{N}}=b_{(j \bmod N)}\right)$, and $b_{i} \leq \sqrt{P /\left(\left|g_{i}\right|^{2} P+\sigma_{w}^{2}\right)}(0 \leq i<N)$ represents the amplification factor of the $i$-th relay.

Suppose that $\rho$ goes to infinity, $R$ grows according to $r \log \rho$ ( $r$ is the multiplexing gain in DMT), we shall derive a series of TRT theorems for the SAF protocol under different scenarios. We firstly focus on the scenario $l-1>N$, where we consider two cases, i.e., $(l-1)_{N}=0$ and $(l-1)_{N} \neq 0$. Then we further discuss the scenario $l-1 \leq N$.

\section{A. Case 1: $(l-1)_{N}=0$}

For this case, we have a theorem as follows.

Theorem 1: We consider a $1-N-1$ relaying network with the SAF protocol. The frame length of the transmitted signals at the source is $l$. If the frame length $l$ satisfies the following conditions: $l \geq N+1$ and $(l-1)_{N}=0$, then the TRT of the $1-N-1$ relaying network with the SAF protocol can be expressed as

$$
\lim _{\substack{\rho \rightarrow \infty \\(R, \rho) \in \mathcal{R}(k)}} \frac{\log P_{o}(R, \rho)-c(k) R}{\log \rho}=-g(k),
$$

where $k$ is a non-negative integer, representing the index of an operating region and $0 \leq k \leq N, g(k)$ is referred to as the reliability gain coefficient, and $\frac{g(k)}{c(k)}$ is referred to as the throughput gain coefficient. In (8), $\mathcal{R}(k)$ means the $k$-th operating region, $0 \leq k \leq N$, specified by

$$
\begin{aligned}
& \mathcal{R}(k) \triangleq \\
& \begin{cases}\left\{(R, \rho) \mid \frac{(l-1)(k+1)}{l N}>\frac{R}{\log \rho}>\frac{(l-1) k}{l N}\right\}, & 0 \leq k<N, \\
\left\{(R, \rho) \mid 1>\frac{R}{\log \rho}>\frac{l-1}{l}\right\}, & k=N .\end{cases}
\end{aligned}
$$

In each operating region in (9), $\{c(k), g(k)\}$ are accordingly defined as

$$
\{c(k), g(k)\} \triangleq \begin{cases}\left\{1+\frac{l N}{l-1}, 1+N\right\}, & 0 \leq k<N \\ \{1,1\}, & k=N .\end{cases}
$$

Proof: Please refer to Appendix B.

Here, we would like to have a discuss about the practical implications of the TRT results. The reliability gain coefficient $g(k)$ represents the slope of the outage probability curve. A larger $g(k)$ implies a steeper curve, and thus more releasibility. The throughput gain coefficient $\frac{g(k)}{c(k)}$ implies that to keep the outage probability $P_{o}$ constant, the variations of the rate and the SNR must satisfy $\Delta R \approx \frac{g(k)}{c(k)} \Delta \log \rho$ in the operating region $\mathcal{R}(k)$. That is, by increasing the logarithmic SNR by $\Delta \log \rho$, we can obtain $\frac{g(k)}{c(k)} \Delta \log \rho$ extra rate while keeping the same outage probability. 


$$
\begin{gathered}
\mathcal{R}_{1}(k) \triangleq \begin{cases}\left\{(R, \rho) \mid \frac{(l-1+N-m)(k+1)}{l N}>\frac{R}{\log \rho}>\frac{(l-1+N-m) k}{l N}\right\}, & 0 \leq k<m, \\
\left.(R, \rho) \mid \frac{(l-1-m)(k+1)+m N}{l N}>\frac{R}{\log \rho}>\frac{(l-1-m) k+m N}{l N}\right\}, & m \leq k<N, \\
\left.(R, \rho) \mid 1>\frac{R}{\log \rho}>\frac{l-1}{l}\right\}, & k=N\end{cases} \\
\mathcal{R}_{2}(k) \triangleq \begin{cases}\left\{(R, \rho) \mid \frac{(l-1-m)(k+1)}{l N}>\frac{R}{\log \rho}>\frac{(l-1-m) k}{l N}\right\}, & 0 \leq k<N-m, \\
\left.(R, \rho) \mid \frac{(l-1+N-m)(k+1)+m N-N^{2}}{l N}>\frac{R}{\log \rho}>\frac{(l-1+N-m) k+m N-N^{2}}{l N}\right\}, & N-m \leq k<N, \\
\left.(R, \rho) \mid 1>\frac{R}{\log \rho}>\frac{l-1}{l}\right\}, & k=N\end{cases}
\end{gathered}
$$

\section{B. Case 2: $(l-1)_{N} \neq 0$}

We now consider a more general case as $(l-1)_{N}=m, m \neq$ 0 . That is, after the source's symbols are equally scheduled to the $N$ relays, there are still $m$ symbols remained to be scheduled. We can utilize the prearranged relays (by roundrobin scheduling) to forward the remained $m$ symbols. Also, we can randomly choose $m$ relays to forward the remained $m$ symbols. It is obvious that these two transmission schemes have the same TRT expressions as that in Theorem 1.

In addition, we are interested in another two extreme schemes. One extreme scheme is that the remaining $m$ symbols are transmitted through the best $m$ source-relaydestination channels. The other extreme scheme is that the $m$ symbols are transmitted through the worst $m$ source-relaydestination channels. As discussed in Appendix B, the channel quality of the $i$-th source-relay-destination channel can be represented by $\alpha_{i}+\beta_{i}$, where $\alpha_{i}$ and $\beta_{i}$ are defined in Equation (45). Thus, we can differentiate the qualities of the $N$ source-relay-destination channels by comparing the values of $\alpha_{0}+\beta_{0}, \alpha_{1}+\beta_{1}, \cdots, \alpha_{N-1}+\beta_{N-1}$. Regarding the TRT expressions of the two extreme schemes, we have the following theorem.

Theorem 2: In the case where $(l-1)_{N}=m, m \neq 0$, two extreme schemes are taken into consideration. One scheme is that the best $m$ source-relay-destination channels are used (BCU) to transmit the remaining $m$ symbols. The operating regions in this scheme are shown in (11), and $\left\{c_{1}(k), g_{1}(k)\right\}$ are defined according to $\mathcal{R}_{1}(k)$, that is,

$$
\begin{aligned}
& \left\{c_{1}(k), g_{1}(k)\right\} \triangleq \\
& \begin{cases}\left\{1+\frac{l N}{l-1+N-m}, 1+N\right\}, & 0 \leq k<m, \\
\left\{1+\frac{l N}{l-1-m}, 1+\frac{(l-1) N}{l-1-m}\right\}, & m \leq k<N, \\
\{1,1\}, & k=N .\end{cases}
\end{aligned}
$$

The other scheme is that the worst $m$ source-relaydestination channels are used (WCU) to transmit the remaining $m$ symbols. Then the operating regions are given by (12), and $\left\{c_{2}(k), g_{2}(k)\right\}$ are defined according to $\mathcal{R}_{2}(k)$, that is,

$$
\begin{aligned}
& \left\{c_{2}(k), g_{2}(k)\right\} \triangleq \\
& \begin{cases}\left\{1+\frac{l N}{l-1-m}, 1+N\right\}, & 0 \leq k<N-m, \\
\left.1+\frac{l N}{l-1+N-m}, 1+\frac{(l-1) N}{l-1+N-m}\right\}, & N-m \leq k<N, \\
\{1,1\}, & k=N .\end{cases}
\end{aligned}
$$

Proof: Please refer to Appendix C.
We note that the two extreme schemes in Theorem 2 are not practical as they need full channel information at the transmitter side. Nevertheless, these two schemes are useful and can provide more insights on the TRT of the SAF protocol when considering the relation between $l$ and $N$. For example, consider a scenario where $l$ is much larger than $N$, and we can see that the TRT expressions of both extreme schemes in Theorem 2 can be degraded to the TRT expressions in Theorem 1. In the following subsection, we will further investigate the TRT for the two extreme schemes in the scenario $l \leq N$.

\section{Case 3: $l \leq N$}

We will consider the scenario where the frame length $l$ is no larger than the number of the relays $N$. Then we have $l \leq N$ and $l-1=m$. In this scenario, we use $l-1$ relays to forward the information and there are $N-(l-1)$ relays not being used. If the $l-1$ relays are prearranged or randomly chosen (We denote this scheme as random channels usage (RCU)), then the TRT results in Theorem 1 are still valid. On the other hand, if we choose the BCU (or WCU) scheme, where the first $l-1$ symbols in the frame are forwarded through the best (or worst) $l-1$ relay channels, then Theorem 2 should be revised by taking the relation $m=l-1$ into account. The revised theorem is written as follows.

Theorem 3: When $l \leq N$ and $m=l-1, m \neq 0$, we replace $m$ with $l-1$. Then the operating regions in the BCU scheme are

$$
\mathcal{R}_{1}(k) \triangleq \begin{cases}\left\{(R, \rho) \mid \frac{k+1}{l}>\frac{R}{\log \rho}>\frac{k}{l}\right\}, & 0 \leq k<l-1, \\ \left\{(R, \rho) \mid 1>\frac{R}{\log \rho}>\frac{l-1}{l}\right\}, & k=l-1,\end{cases}
$$

and $\left\{c_{1}(k), g_{1}(k)\right\}$ are defined according to $\mathcal{R}_{1}(k)$, that is,

$$
\left\{c_{1}(k), g_{1}(k)\right\} \triangleq \begin{cases}\{1+l, 1+N\}, & 0 \leq k<l-1, \\ \{1,1\}, & k=l-1 .\end{cases}
$$

On the other hand, the operating regions in the WCU scheme are given by

$$
\mathcal{R}_{2}(k) \triangleq \begin{cases}\left\{(R, \rho) \mid \frac{k+1}{l}>\frac{R}{\log \rho}>\frac{k}{l}\right\}, & 0 \leq k<l-1, \\ \left\{(R, \rho) \mid 1>\frac{R}{\log \rho}>\frac{l-1}{l}\right\}, & k=l-1,\end{cases}
$$

and $\left\{c_{2}(k), g_{2}(k)\right\}$ are defined according to $\mathcal{R}_{2}(k)$, that is,

$$
\left\{c_{2}(k), g_{2}(k)\right\} \triangleq \begin{cases}\{1+l, l\}, & 0 \leq k<l-1, \\ \{1,1\}, & k=l-1 .\end{cases}
$$


Proof: Please refer to Appendix D.

From Theorem 1 and Theorem 3 we can see that the RCU, BCU and WCU schemes share the same divisions of operating regions (Note that the RCU has $N$ operating regions while the BCU and the WCU has $l$ operating regions, but they are essentially the same). We also see that the reliability gain coefficients of the BCU and RCU schemes are the same, while the reliability gain coefficients of the BCU and WCU schemes are different in the operating regions indexed by $k$ $(0 \leq k<l-1)$, i.e., $g_{1}(k)=1+N$ and $g_{2}(k)=l$. Note that the reliability gain coefficient represents the diversity gain in the corresponding operating region. Therefore, the maximum diversity gain of the RCU and BCU schemes achieves $N+1$, while the maximum diversity gain of the WCU scheme is $l$.

We further investigate the throughput gain among the three schemes, which is defined as $t(k) \triangleq \frac{g(k)}{c(k)}$. Note that

$$
\Delta R \approx \frac{g(k)}{c(k)} \Delta \log \rho
$$

The physical meaning of the throughput gain is that if we increase the logarithmic SNR by $\Delta \log \rho$, how much extra rate (throughput) $\Delta R$ we can obtain without reducing the error performance. According to Theorem 1, in the RCU scheme,

$$
t_{R}(k) \triangleq \frac{g(k)}{c(k)}=\frac{1+N}{1+\frac{l N}{l-1}}
$$

in the operating regions indexed by $k(0 \leq k<N)$. According to Theorem 3, in the BCU scheme, we have

$$
t_{B}(k) \triangleq \frac{g_{1}(k)}{c_{1}(k)}=\frac{1+N}{1+l},
$$

in the operating regions indexed by $k(0 \leq k<l-1)$, and in the WCU scheme, we have

$$
t_{W}(k) \triangleq \frac{g_{2}(k)}{c_{2}(k)}=\frac{l}{1+l},
$$

in the operating regions indexed by $k(0 \leq k<l-1)$. Since $l-1<N$, it is clear that $t_{B}(k)>t_{W}(k)>t_{R}(k)$. This means that if we increase the logarithmic SNR by $\Delta \log \rho$, the BCU scheme can obtain the largest throughput growth, while the RCU scheme gets the least the throughput growth. That is to say, the BCU scheme has a largest throughput gain and the RCU scheme has the smallest throughput gain among the three schemes. It is interesting to find out that using the WCU scheme gets larger throughput gain than using the RCU scheme does. An intuitive explanation is that the WCU has a lower diversity gain $l$, and thus has a bit larger throughput gain compare with RCU. Based on above discussions, we have the following remark.

Remark 1: When the frame length $l$ is no larger than the number of the relays $N$, i.e., $l \leq N$, the $R C U$ and $B C U$ schemes outperform the WCU scheme in terms of the reliability gain as both the $R C U$ and $B C U$ schemes can achieve the maximum $N+1$ order diversity gain, while the $W C U$ scheme can only achieve l order diversity gain. Also, the BCU scheme has the largest throughput gain and the $R C U$ scheme has the smallest throughput gain among the three schemes.

\section{TRT THEOREMS FOR THE NAF PROTOCOL}

Now we consider the TRT expressions in the NAF protocol. We first assume that $l_{2 N}=0$. During a frame period, the received signal vector at the destination is

$$
\mathbf{y}=\mathbf{H} \mathbf{x}+\boldsymbol{\Xi} \mathbf{w}+\mathbf{v}
$$

where $\mathbf{y}=\left[y_{0}, \cdots, y_{l-1}\right]^{T}$ represents the length- $l$ vector of the received signals at the destination, $\mathbf{x}=\left[x_{0}, \cdots, x_{l-1}\right]^{T}$ represents the length- $l$ vector of source symbols, w represents the length- $l$ vector of noise samples observed by the relays, and $\mathbf{v}$ represents the length- $l$ vector of noise samples observed by the destination. The $l \times l$ matrix $\Xi$ and the $l \times l$ matrix $\mathbf{H}$ can be obtained according to the scheduling strategy of the NAF protocol. Regarding the TRT of the NAF protocol, we have the following theorem.

Theorem 4: We consider a $1-N-1$ relaying network with the NAF protocol. The frame length of the transmitted signals at the source is $l$. If the frame length $l$ satisfies the following conditions: $l \geq N$ and $l_{2 N}=0$, then the TRT of the $1-N-1$ relaying network with the NAF protocol can be expressed as Equation (8), where the operating regions are given by

$$
\mathcal{R}(k) \triangleq \begin{cases}\left\{(R, \rho) \mid \frac{k+1}{2 N}>\frac{R}{\log \rho}>\frac{k}{2 N}\right\}, & 0 \leq k<N \\ \left.(R, \rho) \mid 1>\frac{R}{\log \rho}>\frac{1}{2}\right\}, & k=N .\end{cases}
$$

In each operating region, $\{c(k), g(k)\}$ are accordingly defined as

$$
\{c(k), g(k)\} \triangleq \begin{cases}\{1+2 N, 1+N\}, & 0 \leq k<N \\ \{1,1\}, & k=N .\end{cases}
$$

Proof: Please refer to Appendix E.

By comparing Theorem 4 with Theorem 1, we can see that the TRT expressions of the two protocols are very similar. If we replace the coefficient $l-1$ in the TRT results of the SAF protocol with $\frac{l}{2}$, we get the TRT results of the NAF protocol. An intuitive explanation is that in the SAF protocol, the relays forward $(l-1)$ symbols of a length- $l$ frame, while in the NAF protocol, the relays only forward half of a length- $l$ frame. In this sense, we may guess the TRT expressions for a general AF protocol (inter-relay interference is not considered), where there are $q(0<q<l)$ symbols in a frame forwarded by the relays. We can replace the $l-1$ in the TRT results of the SAF protocol with $q$ to obtain the TRT expressions for this general AF protocol. For the case when $l_{2 N}=m,(m \neq 0)$ in the NAF protocol, we can directly obtain its TRT expressions from Theorem 2. So we do not repeat the result here.

\section{The Connection Between TRT And DMT}

Now we investigate the relationship between TRT and DMT in the SAF protocol and the NAF protocol. Note that in MIMO systems, the relationship between DMT and TRT is [14]

$$
g(k)=d(k)-k d^{\prime}(k) \text {, and } c(k)=-d^{\prime}(k) .
$$

The DMT expression in the SAF protocol is given by [17] as

$$
d(r)=(1-r)^{+}+N\left(1-\frac{l}{l-1} r\right)^{+} .
$$


It is obvious that in the SAF protocol, the larger the frame length $l$ is, the higher diversity gain can be obtained. The DMT formulation proves that the SAF protocol can get the largest diversity gain. Therefore, the frame length $l$ is chosen to approach the maximum of $d(r)$ as long as the channels permit. Meanwhile, the DMT expression in the NAF protocol is given by [4] as

$$
d(r)=(1-r)^{+}+N(1-2 r)^{+} .
$$

From the DMT curve of both the SAF protocol and the NAF protocol, we can see that the curve is not continuous in the first order differential coefficient, i.e., there are inflexions at the curve. We observe that these inflexions split the DMT curve into segments, which correspond to the operating regions in TRT expressions. Mathematically, by comparing the TRT expressions in Theorem 1 with (27), and by comparing the TRT expressions in Theorem 4 with (28), we find that the relation between TRT and DMT in both the SAF and NAF protocols also satisfies (26) as that in MIMO systems.

Generally speaking, the two AF protocols imitate the processes of MIMO systems. For the two AF protocols, in $\mathcal{R}(N)$, it can be seen as an SISO system, while in $\mathcal{R}(k<N)$, it imitates a MISO system. So the multiplexing gain can not exceed 1 . The slope of each segmented line represents the reliability gain coefficient $g(k)$, which corresponds to the largest diversity gain of $\mathcal{R}(k)$. Realizing that $\Delta \log \rho \approx \frac{c(k)}{g(k)} \Delta R$, the horizontal spacing of outage curves with a $\Delta R$ difference can be worked out. It is related to $N$ and $k$ in the two protocols. Particularly in the SAF protocol, horizontal spacing is also related to the frame length $l$.

\section{Numerical RESUlts AND Simulations}

We investigate the TRT results in a two-relay scenario $(N=$ 2 ) with the SAF protocol. Since the NAF protocol has the similar TRT expressions as the SAF protocol has, we will skip the simulations of the NAF protocol here. We assume that all the channel coefficients are Rayleigh distributed with unit variance, and that noises at all the receivers are Gaussian distributed with the same variance.

From TRT expressions in the two theorems, we find that in addition to $R$ and $\rho$, frame length $l$ is also an important factor of $P_{o}(R, \rho)$. In the simulations, we first show the impact of different frame lengths on the outage curves. Then we utilize the TRT formulations to analyze the relation between the triplets of $R, P_{o}$ and $\rho$ and explore the asymptotic trends of the triplets when $\rho$ goes to infinity. Finally, we compare the TRT predicted outage curves with Monte-Carlo simulations.

Note that the TRT is used to reveal the interplay between SNR, $R$, and $P_{o}$ when SNR goes to infinity. The higher the rate, the higher the SNR at which interesting behaviors manifest themselves. In order to show the validity of our analysis at different rates, as well as to create separation between various curves related to different values of the frame length $l$, a large range of rates are simulated and therefore the range of SNRs shown in the figures are larger than encountered in practical channels, purely for demonstration purposes. Here, we consider the rates as $R=18 \mathrm{bpcu}, 28 \mathrm{bpcu}$ and $38 \mathrm{bpcu}$, and the SNR region from $40 \mathrm{~dB}$ to $180 \mathrm{~dB}$.

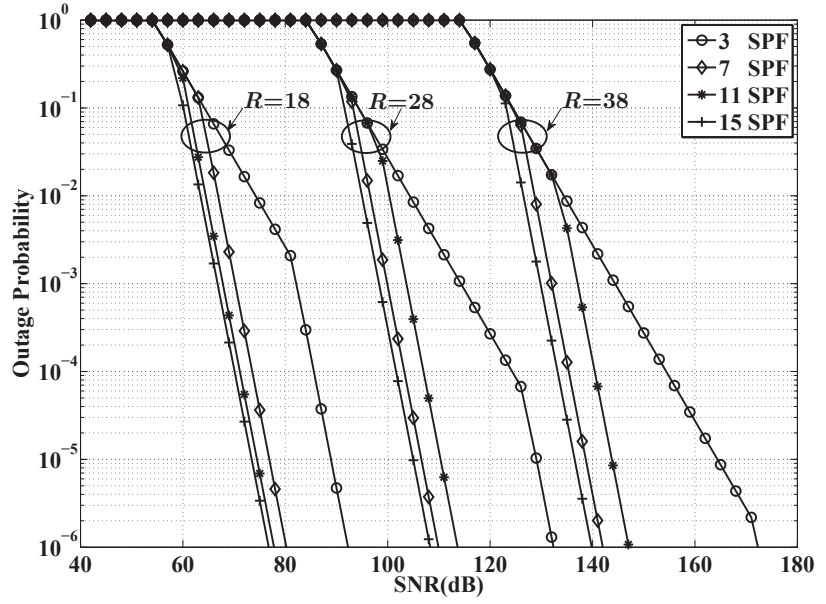

Fig. 4. Outage probability predicted by TRT formulation. Transmission rates are $R=18,28$ and 38 bpcu. For each transmission rate, frame lengths of $l=3,7,11$ and 15 symbols are considered.

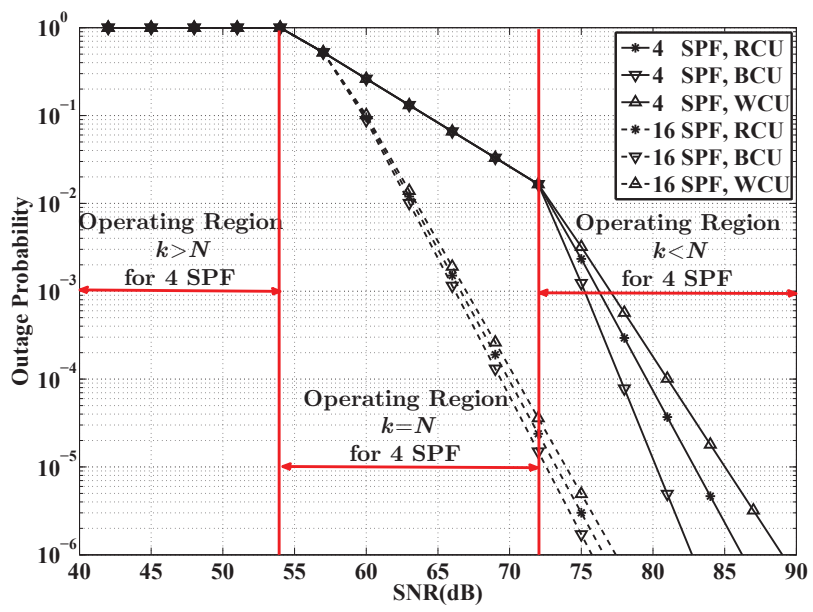

Fig. 5. Outage probability predicted by TRT formulation with transmission rate $R=18$ bpcu. Frame lengths $l=4$ and 16 are considered. For the both frame lengths, we have $(l-1)_{N} \neq 0$. Therefore, the TRT predicted outage curves of RCU, BCU and WCU schemes are considered for the two frame lengths, respectively. Operating regions for frame length 4 are marked in the figure.

To begin with, we focus on the impact of frame length on the outage curves. TRT formulations predict the outage in the SAF protocol as

$\log P_{o}^{T R T}(R, \rho) \approx c(k) R-g(k) \log \rho \quad$ for $\quad(R, \rho) \in \mathcal{R}(k)$.

In the SAF protocol, one out of $l$ symbols in a frame cannot be protected by the relays. Intuitively, large frame length leads to better outage performance. For $(l-1)_{N}=0$, Fig. 4 shows the TRT predicted outage curves under different frame lengths, i.e., $l=3,7,11,15$ symbols per frame (labeled as 'SPF' in all the figures). For $(l-1)_{N} \neq 0$, Fig. 5 shows the TRT predicted outage curves for frame lengths 4 and 16 with transmission rate $R=18$. For the both figures, operating regions can be easily found from the TRT predicted outage curves. The regions of $k>N$ can be seen as outage region, in which the outage always equals to 1 . In the operating region $k=N$, 


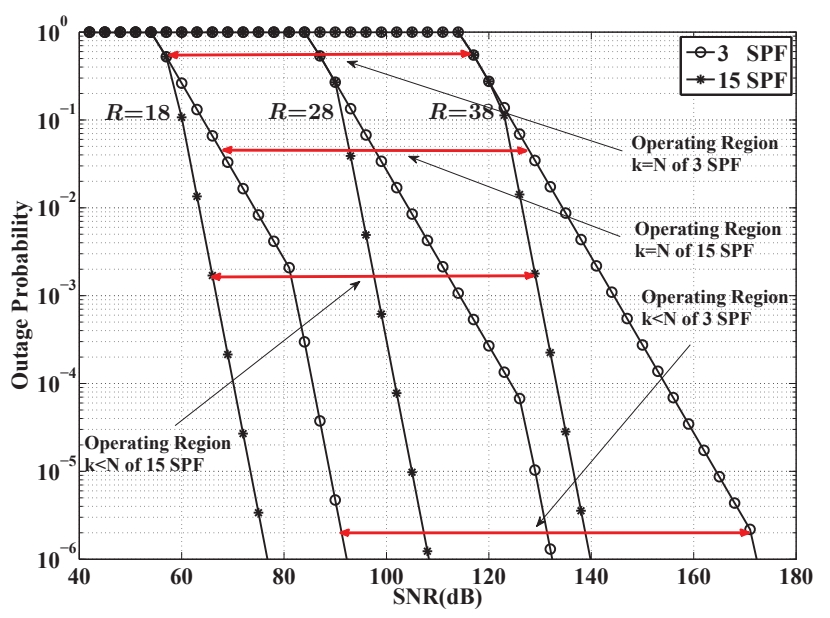

Fig. 6. Outage probability predicted by TRT formulation with frame lengths $l=3$ and 15 . Transmission rates are $R=18,28$ and 38 bpcu. Operating regions are marked for both lengths to explain the trends of outage curves as $R$ and $\rho$ approach infinity. In operating region $k=N$ for frame length 3 , when increasing the rate with $\triangle R=10$, we need to increase the SNR with $\triangle \rho=30 \mathrm{~dB}$ to keep the same outage probability. This is the same for the frame length 15 in the operating region $k=N$. In operating region $k<N$ for frame length 3 , when increasing the rate with $\triangle R=10$, we need to increase the SNR with $\triangle \rho=31.43 \mathrm{~dB}$ to keep the same outage probability. In operating region $k<N$ for frame length 15 , when increasing the rate with $\triangle R=10$, we need to increase the SNR with $\triangle \rho=40 \mathrm{~dB}$ to keep the same outage probability.

the diversity gain is 1 . In the operating region $k<N$, the diversity gain is $N+1=3$. According to Theorem 1 , the operating region $k=N$ is defined as $1>\frac{R}{\log \rho}>\frac{l-1}{l}$. It is obvious that a large frame length $l$ has a small span of operating region $k=N$. The outage curves can rapidly go through the operating region $k=N$ and reach the operating region $k<N$ where the diversity gain is 3 . Therefore, larger frame length leads to a better outage performance. On the other hand, when $l$ is large enough, the advantage due to large frame length becomes less. This is because $\frac{l-1}{l}$ almost keeps the same value as $l$ is large enough and the regimes of the operating region $k=N$ are almost the same for large lengths. Both Fig. 4 and Fig. 5 show that larger frame length leads to better OP performance.

From Fig. 4, we can see that the gap of outage curves between frame lengths 3 and 7 is much bigger than that between frame lengths 11 and 15. Fig. 5 shows the TRT predicted outage curves of transmission rate $R=18$ and $(l-1)_{N}=1$. We consider three transmission schemes for the remained symbol, i.e., random channel usage (RCU), the best channel usage (BCU), and the worst channel usage (WCU). In the RCU scheme, the operating regions are derived according to Theorem 1, i.e., $k>N, k=N$ and $k<N$. In both the BCU scheme and the WCU scheme, according to Theorem 2, the operating regions are $k>N, k=N, 1 \leq k<N$ and $k<1$. The three schemes have the same performance (1order diversity) in the operating regions $k>N$ and $k=N$. With the RCU scheme, the system achieves 3-order diversity in the operating region $k<N$. With the BCU scheme, the system achieves $\left(1+\frac{l-1}{l-2} N\right)$-order diversity in the operating region $1 \leq k<N$ and achieves 3-order diversity in the

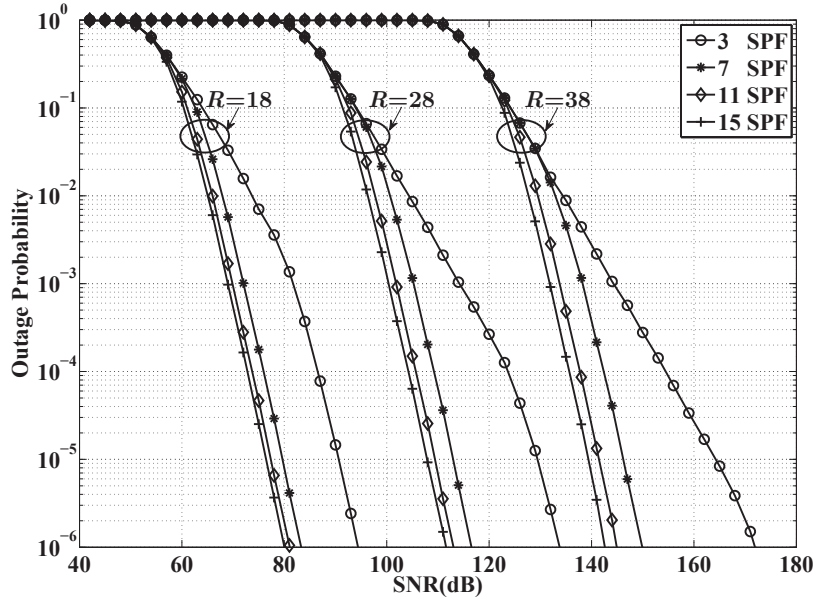

Fig. 7. Infinity SNR outage probability based on Monte-Carlo Simulation. Transmission rates are $R=18 \mathrm{bpcu}, 28 \mathrm{bpcu}$ and $38 \mathrm{bpcu}$. For each transmission rate, frame lengths $l=3,7,11$ and 15 symbols are considered.

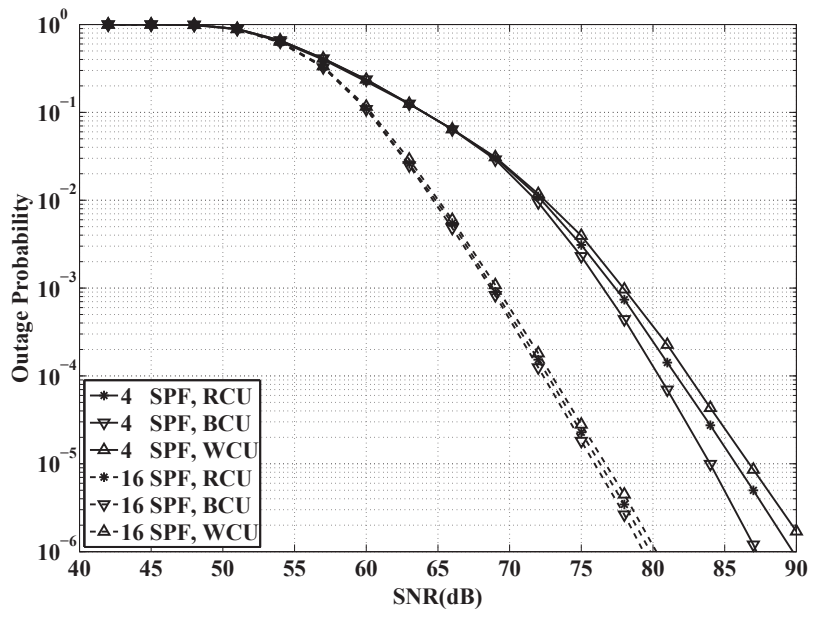

Fig. 8. Infinite SNR outage probability predicted by TRT formulation with transmission rate $R=18 \mathrm{bpcu}$. Frame lengths $l=4$ and 16 are considered. For the both frame lengths, we have $(l-1)_{N} \neq 0$. Therefore, the TRT predicted outage curves of RCU, BCU and WCU schemes are considered for the two frame lengths, respectively.

operating region $k=0$. With the WCU scheme, the system achieves $\left(1+\frac{l-1}{l} N\right)$-order diversity in the operating region $1 \leq k<N$ and achieves 3-order diversity in the operating region $k=0$. Due the space limitation in Fig. 5, the figure shows the operating regions $k \geq 1$. We can see from the figure that, for frame length 4 , when $1 \leq k<N$, the diversity gain of the BCU scheme is 4 -order and the diversity gain of the WCU scheme is 2.5 -order. For frame length 16 , when $1 \leq k<N$, the diversity gain of the BCU scheme is $\frac{22}{7}$-order and the diversity gain of the WCU scheme is $\frac{23}{8}$-order.

Then we investigate the relation between the triplets of $R$, $P_{o}$ and $\rho$ and explore the asymptotic trends of the three variables when $\rho$ goes to infinity. Fig. 6 shows the relation between the SNR increase $\Delta \rho(\mathrm{dB})$ and the rate increase $\Delta R$. Note that in the operating region $k$, we have $\Delta \log \rho \approx \frac{c(k)}{g(k)} \Delta R$, and thus we obtain $\Delta \rho \approx \frac{c(k)}{g(k)} \cdot 3 \Delta R$. For frame length of 3 symbols, in the operating region $k=N$, we have $\Delta \rho \approx 3 \Delta R$, 


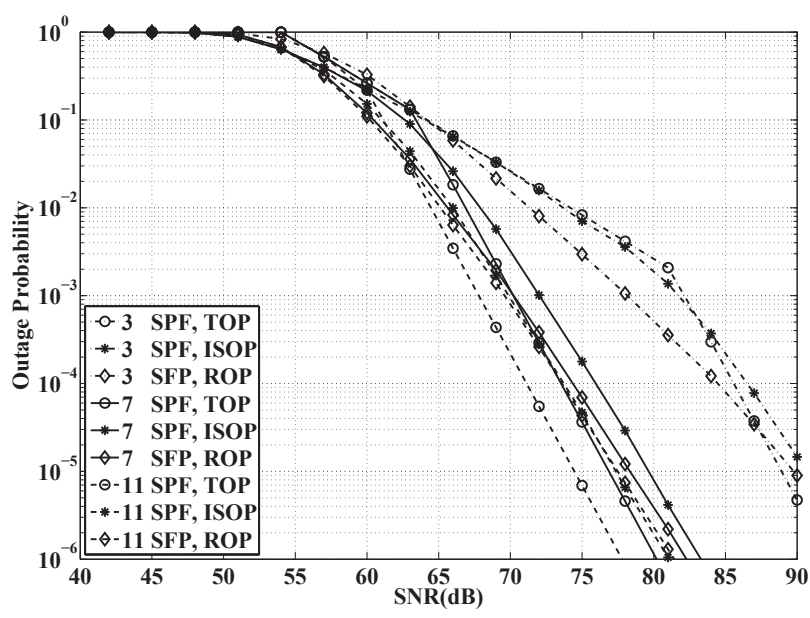

Fig. 9. Comparison of the three kind of outage curves, i.e., the TRT predicted outage (TOP), the infinite SNR outage (ISOP) and the real value of outage (ROP) of transmission rate $R=18$ with frame lengths $l=3,7$ and 11 symbols.

and in the operating region $k<N$, we have $\Delta \rho \approx 4 \Delta R$. For frame length 15 , in the operating region $k=N$, we have $\Delta \rho \approx 3 \Delta R$, and in the operating region $k<N$, we have $\Delta \rho \approx 3.143 \Delta R$. From Fig. 6, we can see the relations between $\Delta \rho$ and $\Delta R$ for the two frame lengths in different operating regions.

In the following, we study the outage curves by MonteCarlo simulations. We introduce two Monte-Carlo based outage expressions. The first one is the real value of outage, denoted as $P_{o}(R, \rho)$. The second one is infinite SNR outage, which is defined as

$$
\begin{aligned}
& P_{o}^{\infty}(R, \rho) \triangleq \lim _{\rho \rightarrow \infty} P_{o}(R, \rho)= \\
& \operatorname{Pr}\left\{\frac{1}{R} \log \left(\left(1+\rho|\hbar|^{2}\right)^{l}+\prod_{j=0}^{l-2}\left(1+\rho\left|g_{j_{N}} h_{j_{N}}\right|^{2}\right)\right)<l\right\} .
\end{aligned}
$$

Actually, the TRT expressions are derived based on $P_{o}^{\infty}(R, \rho)$. Fig. 7 shows the infinite SNR outage curves of frame lengths $l=3,7,11$ and 15 symbols. Compared $P_{o}^{\infty}(R, \rho)$ with the TRT based outage in Fig. 4, we can see that TRT accurately predicts the infinite SNR outage. Fig. 8 shows the infinite SNR outage curves of transmission rate $R=18$ with frame lengths $l=4$ and 16. Also, TRT predicts the infinite SNR outage of the RCU scheme, the BCU scheme and the WCU scheme precisely. Fig. 9 compares the three kind of outage curves, i.e., the TRT predicted outage (TOP), the infinite SNR outage (ISOP) and the real value of outage (ROP) of transmission rate $R=18$ with frame lengths $l=3,7$ and 11 symbols. We can see from the figure that when $\rho$ is large enough, the three kind of outage curves are almost the overlapped with each other. Therefore, we conclude that our TRT expressions precisely reveals the relation and the asymptotic trends between $\rho, R$ and $P_{o}(R, \rho)$ when $\rho$ goes to infinity.

\section{CONCLUSION}

Following Azarian and El Gamal's formulation of throughput vs. reliability in point-to-point MIMO systems, this work analyzes the amplify-forward protocols in a relay channel to reveal the general relationship between $R, \log \rho$ and $P_{o}(R, \rho)$. We derive the TRT formulations of both the SAF protocol and the NAF protocol. We conclude that the relationship between TRT and DMT in MIMO systems carries over to the two AF protocols. We study the issue of the impact of frame length on the $P_{o}(R, \rho)$. Simulations confirm our derivation of the TRT expressions in the AF protocols.

\section{APPENDIX A}

PROOF OF LEMMA 1

The PDFs of $\left|g_{i}\right|^{2}$ and $\left|h_{i}\right|^{2}$ are $\exp \left(-\left|g_{i}\right|^{2}\right)$ and $\exp \left(-\left|h_{i}\right|^{2}\right)$, respectively. By viewing $\left|g_{i} h_{i}\right|^{2}$ as a variable $z$, the PDF of $z$ is $p(z)=p\left(\left|g_{i} h_{i}\right|^{2}\right)=p\left(\left|g_{i}\right|^{2}\right) p\left(\left|h_{i}\right|^{2}\right)=$ $\exp \left\{-\left(\left|g_{i}\right|^{2}+\left|h_{i}\right|^{2}\right)\right\}$. Then we have $p\left(\omega_{i}\right)=p(z) \frac{\mathrm{d} z}{\mathrm{~d} \omega_{i}}=$ $\exp \left\{-\left(\left|g_{i}\right|^{2}+\left|h_{i}\right|^{2}\right)\right\} \frac{R \ln 2}{\rho} 2^{\omega_{i} R}$. Also, since $\mu_{i}+v_{i}=$ $\log \left(\left(\rho\left|g_{i}\right|^{2}\right)\left(\rho\left|h_{i}\right|^{2}\right)\right) / R$, we have $\omega_{i}=\mu_{i}+v_{i}-\frac{\log \rho}{R}$. Then we obtain $p\left(\omega_{i}\right)=\frac{R \ln 2}{\rho} \cdot 2^{\left(\mu_{i}+v_{i}-\frac{\log \rho}{R}\right) R} \exp \left\{-\frac{2^{\mu_{i} R}+2^{v_{i} R}}{\rho}\right\}$. So we complete the proof.

\section{APPENDIX B \\ PROOF OF THEOREM 1}

We will first find the lower bound and upper bound of $\log P_{o}(R, \rho)$, i.e.,

$$
\begin{gathered}
\liminf _{\substack{\rho \rightarrow \infty \\
(R, \rho) \in \mathcal{R}(k)}} \frac{\log P_{o}(R, \rho)-c(k) R}{\log \rho}, \\
\limsup _{\substack{\rho \rightarrow \infty \\
(R, \rho) \in \mathcal{R}(k)}} \frac{\log P_{o}(R, \rho)-c(k) R}{\log \rho} .
\end{gathered}
$$

The conditional mutual information between the source and the destination is given by

$$
I(\mathbf{x} ; \mathbf{y} \mid \mathbf{H})=\log \left(\operatorname{det}\left(\mathbf{I}_{l}+\mathbf{H} \boldsymbol{\Lambda}_{\mathrm{x}} \mathbf{H}^{\dagger} \boldsymbol{\Lambda}_{\mathrm{n}}^{-1}\right)\right)
$$

Here, $\boldsymbol{\Lambda}_{\mathrm{n}}$ is the auto-covariance matrix of noises. A lower bound on $\max _{\boldsymbol{\Lambda}_{\mathrm{x}}} I(\mathbf{x} ; \mathbf{y} \mid \mathbf{H})$ is obtained by replacing $\boldsymbol{\Lambda}_{\mathrm{x}}$ with $P \mathbf{I}_{l}$, i.e.,

$$
\max _{\boldsymbol{\Lambda}_{\mathrm{x}}} \frac{I(\mathbf{x} ; \mathbf{y} \mid \mathbf{H})}{R} \geq \frac{\log \left(\operatorname{det}\left(\mathbf{I}_{l}+\frac{P}{\sigma_{m}^{2}} \mathbf{H} \mathbf{H}^{\dagger}\right)\right)}{R},
$$

where we define

$$
\sigma_{m}^{2}=\max _{0 \leq i<N}\left\{\left|h_{i} b_{i}\right|^{2}\right\} \sigma_{w}^{2}+\sigma_{v}^{2} .
$$

On the other hand, since $\log (\operatorname{det}(\cdot))$ is an increasing function on the cone of positive-definite Hermitian matrices and since $\lambda_{\max } \mathbf{I}_{l}-\boldsymbol{\Lambda}_{\mathrm{x}} \geq 0$ (where $\lambda_{\max }$ represents the largest eigenvalue of $\left.\boldsymbol{\Lambda}_{\mathbf{x}}\right)$, we obtain the upper bound of $\max _{\boldsymbol{\Lambda}_{\mathrm{x}}} I(\mathbf{x} ; \mathbf{y} \mid \mathbf{H})$ as [14], i.e.,

$$
\max _{\Lambda_{\mathrm{x}}} \frac{I(\mathbf{x} ; \mathbf{y} \mid \mathbf{H})}{R} \leq \frac{\log \left(\operatorname{det}\left(\mathbf{I}_{l}+\frac{\lambda_{\max }}{\sigma_{v}^{2}} \mathbf{H H}^{\dagger}\right)\right)}{R} .
$$

As $\lambda \max$ is of the same exponential order as $P$, the two bounds of $\max _{\boldsymbol{\Lambda}_{\mathbf{x}}} \frac{I(\mathbf{x} ; \mathbf{y} \mid \mathbf{H})}{\log \rho}$ converge as $\rho$ grows to infinity. 
We define

$$
\frac{P}{\sigma_{m}^{2}} \triangleq \tau \rho, \quad \text { and } \quad \frac{\lambda_{\max }}{\sigma_{v}^{2}} \triangleq \kappa \rho .
$$

Then we have $0<\tau<1$ and $1<\kappa<\infty$. According to the [16, Lemma 4], we have

$$
\begin{aligned}
& \operatorname{det}\left(\mathbf{I}_{l}+\rho \mathbf{H} \mathbf{H}^{\dagger}\right)= \\
& \left(\rho|\hbar|^{2}\right)^{l}+\prod_{j=0}^{l-2}\left(1+\rho\left|h_{j_{N}} g_{j_{N}}\right|^{2}\right)+O\left(\left(\rho|\hbar|^{2}\right)^{l-1}\right),
\end{aligned}
$$

where $j_{N}$ represents $j \bmod N$. Then we have

$$
\operatorname{det}\left(\mathbf{I}_{l}+\rho \mathbf{H} \mathbf{H}^{\dagger}\right) \doteq\left(1+\rho|\hbar|^{2}\right)^{l}+\prod_{j=0}^{l-2}\left(1+\rho\left|h_{j_{N}} g_{j_{N}}\right|^{2}\right),
$$

and the two bounds on $\max _{\boldsymbol{\Lambda}_{\mathbf{x}}} \frac{I(\mathbf{x} ; \mathbf{y} \mid \mathbf{H})}{R}$ are

$$
\begin{aligned}
& \lim _{\rho \rightarrow \infty} \max _{\boldsymbol{\Lambda}_{\mathrm{x}}} \frac{I(\mathbf{x} ; \mathbf{y} \mid \mathbf{H})}{R} \geq \\
& \lim _{\rho \rightarrow \infty} \frac{1}{R} \log \left(\left(1+\tau \rho|\hbar|^{2}\right)^{l}+\prod_{j=0}^{l-2}\left(1+\tau \rho\left|g_{j_{N}} h_{j_{N}}\right|^{2}\right)\right), \\
& \lim _{\rho \rightarrow \infty} \max _{\boldsymbol{\Lambda}_{\mathrm{x}}} \frac{I(\mathbf{x} ; \mathbf{y} \mid \mathbf{H})}{R} \leq \\
& \lim _{\rho \rightarrow \infty} \frac{1}{R} \log \left(\left(1+\kappa \rho|\hbar|^{2}\right)^{l}+\prod_{j=0}^{l-2}\left(1+\kappa \rho\left|g_{j_{N}} h_{j_{N}}\right|^{2}\right)\right) .
\end{aligned}
$$

Subsequently, we get the lower bound and the upper bound of $P_{o}(R, \rho)$ respectively as shown in (40) and (41), respectively.

In the following, we will use the above two inequalities to derive the two bounds of (31).

1) Lower Bound: Based on the channel model of the SAF protocol, we define the following variables related to the channel coefficients and the transmission rate as

$$
\begin{gathered}
\bar{\beta} \triangleq \lim _{\rho \rightarrow \infty} \frac{\log \left(1+\kappa \rho|\hbar|^{2}\right)}{R}, \\
\gamma_{i} \triangleq \lim _{\rho \rightarrow \infty} \frac{\log \left(1+\kappa \rho\left|g_{i} h_{i}\right|^{2}\right)}{R},
\end{gathered}
$$

where $0 \leq i<N$. In addition, we define $K_{\kappa} \triangleq$ $R \ln 2 / \kappa$ and $e_{\kappa} \triangleq e^{\frac{1}{\kappa}}$. Now, let us focus on the region

$$
\lim \max \left\{l \log \left(1+\kappa \rho|\hbar|^{2}\right), \sum_{j=0}^{l-2} \log \left(1+\kappa \rho\left|g_{j_{N}} h_{j_{N}}\right|^{2}\right)\right\}<R l .
$$

By replacing the channel coefficients with $\bar{\beta}$ and $\gamma_{i}$ and defining $\gamma \triangleq\left\{\gamma_{0}, \gamma_{1}, \cdots, \gamma_{N-1}\right\}$, we obtain another form of the outage region of $P_{o}(R, \rho)$ 's lower bound as

$$
\mathcal{O}_{\kappa} \triangleq\left\{\bar{\beta} \in \mathbb{R}^{+}, \gamma \in \mathbb{R}^{N+} \mid \bar{\beta}<1, \frac{1}{l} \sum_{j=0}^{l-2} \gamma_{j_{N}}<1\right\} .
$$

From the definition in (42), we can see that $\gamma_{i}$ depends on the channel from the source to $r_{i}$ and the channel from $r_{i}$ to the destination. We further define two variables as

$$
\begin{aligned}
& \alpha_{i} \triangleq \lim _{\rho \rightarrow \infty} \frac{\log \left(1+\kappa \rho\left|g_{i}\right|^{2}\right)}{R}, \\
& \beta_{i} \triangleq \lim _{\rho \rightarrow \infty} \frac{\log \left(1+\kappa \rho\left|h_{i}\right|^{2}\right)}{R} .
\end{aligned}
$$

Since

$$
\left(1+\kappa \rho\left|g_{i}\right|^{2}\right)\left(1+\kappa \rho\left|h_{i}\right|^{2}\right) \doteq 1+\kappa^{2} \rho^{2}\left|g_{i} h_{i}\right|^{2},
$$

we have $\gamma_{i}=\alpha_{i}+\beta_{i}-\frac{\log \kappa \rho}{R}$. In the following, we may represent vector $\boldsymbol{\gamma}$ with $\boldsymbol{\alpha}$ and $\boldsymbol{\beta}$ in some cases, where $\boldsymbol{\alpha}=\left\{\alpha_{0}, \alpha_{1}, \cdots, \alpha_{N-1}\right\}$ and $\boldsymbol{\beta}=\left\{\beta_{0}, \beta_{1}, \cdots, \beta_{N-1}\right\}$. To simplify the following analysis, and also for the reason that the relays are used from $r_{0}$ to $r_{N-1}$ in the round-robin manner, we arrange the channels in terms of relays' connect quality, i.e., $\alpha_{0}+\beta_{0} \geq \alpha_{1}+\beta_{1} \geq \cdots \geq \alpha_{N-1}+\beta_{N-1} \geq 0$. Note that this arrangement is only for the analysis purpose and does not have to be the transmission order.

According to Lemma 1, we obtain the PDF of $\gamma_{i}$ as

$$
\begin{aligned}
& p\left(\gamma_{i}\right)= \\
& \frac{R \ln 2}{\kappa \rho} \cdot 2^{\left(\alpha_{i}+\beta_{i}-\frac{\log \kappa \rho}{R}\right) R} \exp \left\{-\frac{2^{\alpha_{i} R}-1+2^{\beta_{i} R}-1}{\kappa \rho}\right\} .
\end{aligned}
$$

The PDF of vector $(\bar{\beta}, \boldsymbol{\gamma})$ can be written as $p(\bar{\beta}, \boldsymbol{\gamma})=$ $p(\bar{\beta}) p\left(\gamma_{0}\right) \cdots p\left(\gamma_{N-1}\right)$ and

$$
\begin{aligned}
& p(\bar{\beta}, \gamma)=\left(\frac{K_{\kappa}}{\rho}\right)^{N+1} 2^{\bar{\beta} R} 2^{\sum_{i=0}^{N-1}\left(\alpha_{i}+\beta i-\frac{\log \kappa \rho}{R}\right) R} . \\
& \exp \left\{-\frac{2^{\bar{\beta} R}-1}{\kappa \rho}-\sum_{i=0}^{N-1} \frac{2^{\alpha_{i} R}-1+2^{\beta i R}-1}{\kappa \rho}\right\} .
\end{aligned}
$$

According to (45), we can give a more compact form of the joint PDF of $(\bar{\beta}, \gamma)$ as

$$
\begin{array}{r}
p(\bar{\beta}, \boldsymbol{\alpha}, \boldsymbol{\beta})=K_{\kappa}^{N+1} e_{\kappa}^{\frac{2 N+1}{\rho}} \rho^{-(N+1)} 2^{f(\bar{\beta}, \boldsymbol{\alpha}, \boldsymbol{\beta}) R} . \\
\quad \exp \left\{-\frac{2^{\bar{\beta} R}}{\kappa \rho}-\sum_{i=0}^{N-1} \frac{2^{\alpha_{i} R}+2^{\beta_{i} R}}{\kappa \rho}\right\}, \\
\text { where } f(\bar{\beta}, \boldsymbol{\alpha}, \boldsymbol{\beta}) \triangleq \bar{\beta}+\sum_{i=0}^{N-1}\left(\alpha_{i}+\beta_{i}-\frac{\log \kappa \rho}{R}\right) .
\end{array}
$$

Consider (40) gives the lower bound of $P_{o}(R, \rho)$, we have

$$
P_{o}(R, \rho) \geq \iiint_{\mathcal{O}_{\kappa}} p(\bar{\beta}, \boldsymbol{\alpha}, \boldsymbol{\beta}) \mathrm{d} \bar{\beta} \mathrm{d} \boldsymbol{\alpha} \mathrm{d} \boldsymbol{\beta} .
$$

Multiplying both sides with $2^{-c(k) R}$, then we have

$$
P_{o}(R, \rho) 2^{-c(k) R} \geq 2^{-c(k) R} \iiint_{\mathcal{O}_{\kappa}} p(\bar{\beta}, \boldsymbol{\alpha}, \boldsymbol{\beta}) \mathrm{d} \bar{\beta} \mathrm{d} \boldsymbol{\alpha} \mathrm{d} \boldsymbol{\beta} .
$$

We search a subset region $\mathcal{O}_{\kappa, \epsilon_{0}} \subset \mathcal{O}_{\kappa}$ such that

$$
\begin{aligned}
& \mathcal{O}_{\kappa, \epsilon_{0}} \triangleq\left\{(\bar{\beta}, \boldsymbol{\alpha}, \boldsymbol{\beta}) \in \mathcal{O}_{\kappa} \mid \exists \epsilon_{0}>0, \text { s.t. } \frac{\log \rho}{R}-\epsilon_{0} \geq \bar{\beta},\right. \\
& \left.\frac{\log \sqrt{\kappa} \rho}{R}-\frac{\epsilon_{0}}{2} \geq \max \left\{\alpha_{\max }, \beta_{\max }\right\}\right\},
\end{aligned}
$$

where $\alpha_{\max }=\max \left\{\alpha_{0}, \cdots, \alpha_{N-1}\right\}$ and $\beta_{\max }=$ $\max \left\{\beta_{0}, \cdots, \beta_{N-1}\right\}$. Replacing $\bar{\beta}$ with $\left(\frac{\log \rho}{R}-\epsilon_{0}\right)$ and $\alpha_{i}$ 


$$
\begin{aligned}
& \lim _{\rho \rightarrow \infty} P_{o}(R, \rho) \geq \operatorname{Pr}\left\{\lim _{\rho \rightarrow \infty} \frac{1}{R} \log \left(\left(1+\kappa \rho|\hbar|^{2}\right)^{l}+\prod_{j=0}^{l-2}\left(1+\kappa \rho\left|g_{j_{N}} h_{j_{N}}\right|^{2}\right)\right)<l\right\} \\
& \lim _{\rho \rightarrow \infty} P_{o}(R, \rho) \leq \operatorname{Pr}\left\{\lim _{\rho \rightarrow \infty} \frac{1}{R} \log \left(\left(1+\tau \rho|\hbar|^{2}\right)^{l}+\prod_{j=0}^{l-2}\left(1+\tau \rho\left|g_{j_{N}} h_{j_{N}}\right|^{2}\right)\right)<l\right\}
\end{aligned}
$$

and $\beta_{i}, i=1, \cdots, N$, with $\left(\frac{\log \sqrt{\kappa} \rho}{R}-\frac{\epsilon_{0}}{2}\right)$. Then we have

$$
\begin{array}{r}
P_{o}(R, \rho) 2^{-c(k) R} \geq K_{\kappa}^{N+1} e_{\kappa}^{\frac{2 N+1}{\rho}-\sqrt{\kappa} N 2^{1-\frac{\epsilon_{0}}{2} R}-2^{-\epsilon_{0} R}} . \\
\rho^{-(N+1)} 2^{-c(k) R} \iiint_{\mathcal{O}_{\kappa, \epsilon_{0}}} 2^{f(\bar{\beta}, \boldsymbol{\alpha}, \boldsymbol{\beta}) R} \mathrm{~d} \bar{\beta} \mathrm{d} \boldsymbol{\alpha} \mathrm{d} \boldsymbol{\beta} .
\end{array}
$$

Now, we define $\boldsymbol{\zeta}_{\epsilon_{0}}$ as

$$
\boldsymbol{\zeta}_{\epsilon_{0}} \triangleq \arg \sup _{(\bar{\beta}, \boldsymbol{\alpha}, \boldsymbol{\beta}) \in \mathcal{O}_{\kappa, \epsilon_{0}}} f(\bar{\beta}, \boldsymbol{\alpha}, \boldsymbol{\beta}) .
$$

Because of the continuity of function $f$, for some $\epsilon_{1}>0$, there must exist around $\boldsymbol{\zeta}_{\epsilon_{0}}$ a neighborhood $I_{\epsilon_{1}} \subset \mathcal{O}_{\kappa}$, in which $f(\bar{\beta}, \boldsymbol{\alpha}, \boldsymbol{\beta}) \geq f\left(\boldsymbol{\zeta}_{\epsilon_{0}}\right)-\epsilon_{1}$. So in the intersection $\mathcal{O}_{\kappa, \epsilon_{0}} \cap I_{\epsilon_{1}}$, we have

$$
\begin{gathered}
P_{o}(R, \rho) 2^{-c(k) R} \geq K_{\kappa}^{N+1} e_{\kappa}^{\frac{2 N+1}{\rho}-\sqrt{\kappa} N 2^{1-\frac{\epsilon_{0}}{2} R}-2^{-\epsilon_{0} R}} . \\
\rho^{-(N+1)} 2^{\left(f\left(\boldsymbol{\zeta}_{\epsilon_{0}}\right)-c(k) R-\epsilon_{1}\right) R} \operatorname{Vol}\left\{\mathcal{O}_{\kappa, \epsilon_{0}} \cap I_{\epsilon_{1}}\right\},
\end{gathered}
$$

where $\operatorname{Vol}\left\{\mathcal{O}_{\kappa, \epsilon_{0}} \cap I_{\epsilon_{1}}\right\}=\iiint_{\mathcal{O}_{\kappa, \epsilon_{0}} \cap I_{\epsilon_{1}}} \mathrm{~d} \bar{\beta} \mathrm{d} \boldsymbol{\alpha} \mathrm{d} \boldsymbol{\beta}$. Note that all the functions $\operatorname{Vol}\{\cdot\}$ in the following have the similar definition. Then we have

$$
\begin{aligned}
& \frac{\log P_{o}(R, \rho)-c(k) R}{\log \rho} \geq \\
& \frac{\log \left(e_{\kappa}^{\frac{2 N+1}{\rho}-\sqrt{\kappa} N 2^{1-\frac{\epsilon_{0}}{2} R}-2^{-\epsilon_{0} R}} 2^{-\epsilon_{1} R} \operatorname{Vol}\left\{\mathcal{O}_{\kappa, \epsilon_{0}} \cap I_{\epsilon_{1}}\right\}\right)}{\log \rho}+ \\
& \frac{\log K_{\kappa}^{N+1}}{\log \rho}-(N+1)+\left(f\left(\boldsymbol{\zeta}_{\epsilon_{0}}\right)-c(k)\right) \frac{R}{\log \rho} .
\end{aligned}
$$

To obtain $f\left(\boldsymbol{\zeta}_{\epsilon_{0}}\right)$ under the constraint $\mathcal{O}_{\kappa, \epsilon_{0}}$, we partition the operating region according to the value of $\frac{\log \rho}{R}$. Considering a simple situation where $(l-1)_{N}=0$, the operating region $\mathcal{R}_{\delta}(k)$ with $k \in\{0, \cdots, N\}$ can be defined as

$\mathcal{R}_{\delta}(k) \triangleq$

$\begin{cases}\left\{(R, \rho) \mid \frac{1}{\delta}>\frac{\log \rho}{R}>\frac{l N}{l-1}+\delta\right\}, & k=0, \\ \left.(R, \rho) \mid \frac{l N}{(l-1) k}-\delta>\frac{\log \rho}{R}>\frac{l N}{(l-1)(k+1)}+\delta\right\}, & 0<k<N, \\ \left.(R, \rho) \mid \frac{l}{l-1}-\delta>\frac{\log \rho}{R}>1+\delta\right\}, & k=N, \\ \left\{(R, \rho) \mid 1-\delta>\frac{\log \rho}{R}>\delta\right\}, & \text { others, }\end{cases}$

where $\delta$ is an arbitrary small positive value and $\delta \geq \epsilon_{0}$.

Now, we determine the function $f\left(\boldsymbol{\zeta}_{\epsilon_{0}}\right)$ in different operating regions. In the first operating region $\mathcal{R}_{\delta}(0), \frac{\log \rho}{R}$ is so large that it cannot be achieved by $\bar{\beta}, \alpha_{\max }$, and $\beta_{\max }$. Otherwise they will be in $\mathcal{O}_{\kappa}^{c}$. So in such a case, we have

$$
f\left(\boldsymbol{\zeta}_{\epsilon_{0}}\right)=1+\frac{l N}{l-1} .
$$

In the operating region $\mathcal{R}_{\delta}(N)$, all the elements in $\boldsymbol{\alpha}$ and $\boldsymbol{\beta}$ can reach $\frac{\log \sqrt{\kappa} \rho}{R}-\frac{\epsilon_{0}}{2}$ simultaneously while keeping $\bar{\beta}=1$. In this region, we have

$$
f\left(\boldsymbol{\zeta}_{\epsilon_{0}}\right)=1+\left(\frac{\log \rho}{R}-\epsilon_{0}\right) N .
$$

For the case when $k>N$, we have

$$
f\left(\boldsymbol{\zeta}_{\epsilon_{0}}\right)=\left(\frac{\log \rho}{R}-\epsilon_{0}\right)(N+1) .
$$

Finally, we focus on $1<k<N$. In this situation, the supremum of $f\left(\boldsymbol{\zeta}_{\epsilon_{0}}\right)$ occurs at $\bar{\beta}=1$, and

$$
\begin{gathered}
\boldsymbol{\alpha}=\boldsymbol{\beta}=\underbrace{\left(\frac{\log \sqrt{\kappa} \rho}{R}-\frac{\epsilon_{0}}{2}, \cdots, \frac{\log \sqrt{\kappa} \rho}{R}-\frac{\epsilon_{0}}{2}\right.}_{k \text { times }}, \\
\left.\frac{N l}{2(l-1)}+\frac{\log \kappa \rho}{2 R}-\frac{k}{2}\left(\frac{\log \rho}{R}-\epsilon_{0}\right), 0, \cdots, 0\right) .
\end{gathered}
$$

Then Equation (50) becomes $f\left(\boldsymbol{\zeta}_{\epsilon_{0}}\right)=1+\frac{l N}{l-1}$. Considering the values of the function $f\left(\boldsymbol{\zeta}_{\epsilon_{0}}\right)$ in different operating regions, and letting $\delta$ and $\epsilon_{0}$ go to 0 , we get the operating regions $\mathcal{R}(k)$ shown as (9). Consider $f\left(\boldsymbol{\zeta}_{\epsilon_{0}}\right)$ in different operating regions, we have

$$
\begin{aligned}
& \quad \liminf _{\substack{\rho \rightarrow \infty \\
(R, \rho) \in \mathcal{R}(N>k \geq 0)}} \frac{\log P_{o}(R, \rho)-c(k) R}{\log \rho} \geq \\
& -(N+1)+\left(1+\frac{l N}{l-1}-c(k)\right) \times \liminf _{\substack{\rho \rightarrow \infty \\
(R, \rho) \in \mathcal{R}(N>k \geq 0)}} \frac{R}{\log \rho},
\end{aligned}
$$

$$
\begin{aligned}
\liminf _{\substack{\rho \rightarrow \infty \\
(R, \rho) \in \mathcal{R}(k=N)}} \frac{\log P_{o}(R, \rho)-c(k) R}{\log \rho} \geq \\
\quad-1+(1-c(k)) \times \liminf _{\substack{\rho \rightarrow \infty \\
(R, \rho) \in \mathcal{R}(k=N)}} \frac{R}{\log \rho} .
\end{aligned}
$$

2) Upper Bound: We now turn our attention to Equation (41), i.e., the upper bound of $P_{o}(R, \rho)$. We first introduce the following change of variables, i.e.,

$$
\begin{aligned}
& \bar{\beta} \triangleq \lim _{\rho \rightarrow \infty} \frac{\log \left(1+\tau \rho|\hbar|^{2}\right)}{R}, \\
& \gamma_{i} \triangleq \lim _{\rho \rightarrow \infty} \frac{\log \left(1+\tau \rho\left|g_{i} h_{i}\right|^{2}\right)}{R} .
\end{aligned}
$$


We also define $K_{\tau} \triangleq R \ln 2 / \tau$ and $e_{\tau} \triangleq e^{\frac{1}{\tau}}$. In addition, we redefine $\alpha_{i}$ and $\beta_{i}$ in (45) as

$$
\begin{aligned}
& \alpha_{i} \triangleq \lim _{\rho \rightarrow \infty} \frac{\log \left(1+\tau \rho\left|g_{i}\right|^{2}\right)}{R} \\
& \beta_{i} \triangleq \lim _{\rho \rightarrow \infty} \frac{\log \left(1+\tau \rho\left|h_{i}\right|^{2}\right)}{R} .
\end{aligned}
$$

We obtain the outage region of $P_{o}(R, \rho)$ 's upper bound as

$$
\mathcal{O}_{\tau} \triangleq\left\{\bar{\beta} \in \mathbb{R}^{+}, \gamma \in \mathbb{R}^{N+} \mid \bar{\beta}<1, \frac{1}{l} \sum_{j=0}^{l-2} \gamma_{j_{N}}<1\right\} .
$$

We split the outage region into four disjoint subsets as shown in (68). The function $2^{-c(k) R} \iiint p(\bar{\beta}, \boldsymbol{\alpha}, \boldsymbol{\beta}) \mathrm{d} \bar{\beta} \mathrm{d} \boldsymbol{\alpha} \mathrm{d} \boldsymbol{\beta}$ in the four subsets are represented by $O_{-}(R, \rho), O_{ \pm}(R, \rho)$, $O_{\mp}(R, \rho)$, and $O_{+}(R, \rho)$, respectively. Thus we have

$$
\begin{aligned}
& \frac{\log P_{o}(R, \rho)-c(k) R}{\log \rho} \leq \frac{\log O_{+}(R, \rho)}{\log \rho}+ \\
& \frac{\log \left(1+\frac{O_{-}(R, \rho)}{O_{+}(R, \rho)}+\frac{O_{ \pm}(R, \rho)}{O_{+}(R, \rho)}+\frac{O_{\mp}(R, \rho)}{O_{+}(R, \rho)}\right)}{\log \rho} .
\end{aligned}
$$

We redefine

$$
f(\bar{\beta}, \boldsymbol{\alpha}, \boldsymbol{\beta}) \triangleq \bar{\beta}+\sum_{i=0}^{N-1}\left(\alpha_{i}+\beta_{i}-\frac{\log \tau \rho}{R}\right) .
$$

First, in the subset $\mathcal{O}_{\tau,-\epsilon_{0}}$, we have

$$
\begin{aligned}
O_{-}(R, \rho) \leq & K_{\tau}^{N+1} e_{\tau}^{\frac{2 N+1}{\rho}-\sqrt{\tau} 2^{\frac{\epsilon_{0}}{2} R}-2^{\epsilon_{0} R}} \rho^{-(N+1) .} \\
& 2^{-c(k) R} \iiint 2^{f(\bar{\beta}, \boldsymbol{\alpha}, \boldsymbol{\beta}) R} \mathrm{~d} \bar{\beta} \mathrm{d} \boldsymbol{\alpha} \mathrm{d} \boldsymbol{\beta} \\
\leq & K_{\tau}^{N+1} e_{\tau}^{\frac{2 N+\epsilon_{0}}{\rho}-\sqrt{\tau} 2^{\frac{\epsilon_{0}}{2} R}-2^{\epsilon_{0} R}} \rho^{-(N+1) .} \\
& 2^{\left(f\left(\boldsymbol{\zeta}_{-\epsilon_{0}}\right)-c(k)\right) R} \operatorname{Vol}\left\{\mathcal{O}_{\tau}\right\}
\end{aligned}
$$

where $\zeta_{-\epsilon_{0}}$ is defined as

$$
\boldsymbol{\zeta}_{-\epsilon_{0}} \triangleq \arg \sup _{(\bar{\beta}, \boldsymbol{\alpha}, \boldsymbol{\beta}) \in \mathcal{O}_{\tau,-\epsilon_{0}}} f(\bar{\beta}, \boldsymbol{\alpha}, \boldsymbol{\beta}) .
$$

In the similar way, $\boldsymbol{\zeta}_{ \pm \epsilon_{0}}, \boldsymbol{\zeta}_{\mp \epsilon_{0}}$, and $\boldsymbol{\zeta}_{\epsilon_{0}}$ can also be defined. Then in $\mathcal{O}_{\tau}^{ \pm \epsilon_{0}}$ and $\mathcal{O}_{\tau}^{\mp \epsilon_{0}}$, we have

$$
\begin{aligned}
& O_{ \pm}(R, \rho) \leq \\
& K_{\tau}^{N+1} e_{\tau}^{\frac{1}{\rho}-2^{\epsilon_{0} R}} \rho^{-(N+1)} 2^{\left(f\left(\boldsymbol{\zeta}_{ \pm \epsilon_{0}}\right)-c(k)\right) R} \operatorname{Vol}\left\{\mathcal{O}_{\tau}\right\} \\
& O_{\mp}(R, \rho) \leq \\
& K_{\tau}^{N+1} e_{\tau}^{\frac{2 N}{\rho}-\sqrt{\tau} 2^{\frac{\epsilon_{0}}{2} R}} \times \rho^{-(N+1)} 2^{\left(f\left(\boldsymbol{\zeta}_{\mp \epsilon_{0}}\right)-c(k)\right) R} \operatorname{Vol}\left\{\mathcal{O}_{\tau}\right\} .
\end{aligned}
$$

We turn to another two subsets, one is

$$
\begin{gathered}
\mathcal{O}_{\tau, \epsilon_{1}} \triangleq\left\{(\bar{\beta}, \boldsymbol{\alpha}, \boldsymbol{\beta}) \in \mathcal{O}_{\tau} \mid \exists \epsilon_{1}>0, \text { s.t. } \frac{\log \rho}{R}-\epsilon_{1} \geq \bar{\beta},\right. \\
\left.\frac{\log \sqrt{\tau} \rho}{R}-\frac{\epsilon_{1}}{2} \geq \max \left\{\alpha_{\max }, \beta_{\max }\right\}\right\}
\end{gathered}
$$

and the other is $\zeta_{\epsilon_{1}}$ 's neighborhood $I_{\epsilon_{2}}$, in which we have

$$
\begin{array}{r}
O_{+}(R, p) \geq K_{\tau}^{N+1} e_{\tau}^{\frac{2 N+1}{\rho}-\sqrt{\tau} N 2^{1-\frac{\epsilon_{1}}{2} R}-2^{-\epsilon_{1} R}} \rho^{-(N+1) .} \\
2^{\left(f\left(\boldsymbol{\zeta}_{\epsilon_{1}}\right)-c(k) R-\epsilon_{2}\right) R} \operatorname{Vol}\left\{\mathcal{O}_{\tau, \epsilon_{1}} \cap I_{\epsilon_{2}}\right\} .
\end{array}
$$

Then we have

$$
\begin{aligned}
\frac{O_{-}(R, \rho)}{O_{+}(R, \rho)} \leq & e_{\tau}^{\sqrt{\tau} N 2^{1-\frac{\epsilon_{1}}{2} R}-\sqrt{\tau} 2^{\frac{\epsilon_{0}}{2} R}+2^{-\epsilon_{1} R}-2^{\epsilon_{0} R} .} \\
& 2^{\left(f\left(\boldsymbol{\zeta}_{-\epsilon_{0}}\right)-f\left(\boldsymbol{\zeta}_{\epsilon_{1}}\right)+\epsilon_{2}\right) R} \frac{\operatorname{Vol}\left\{\mathcal{O}_{\tau}\right\}}{\operatorname{Vol}\left\{\mathcal{O}_{\tau, \epsilon_{1}} \cap I_{\epsilon_{2}}\right\}}, \\
\frac{O_{ \pm}(R, \rho)}{O_{+}(R, \rho)} \leq & e_{\tau}^{\frac{-2 N}{\rho}+\sqrt{\tau} N 2^{1-\frac{\epsilon_{1}}{2} R}+2^{-\epsilon_{1} R}-2^{\epsilon_{0} R}} \cdot \\
\frac{O_{\mp}(R, \rho)}{O_{+}(R, \rho)} \leq & e_{\tau^{\left(f\left(\boldsymbol{\zeta}_{ \pm \epsilon_{0}}\right)-f\left(\boldsymbol{\zeta}_{\epsilon_{1}}\right)+\epsilon_{2}\right) R} \frac{\operatorname{Vol}\left\{\mathcal{O}_{\tau}\right\}}{\operatorname{Vol}\left\{\mathcal{O}_{\tau, \epsilon_{1}} \cap I_{\epsilon_{2}}\right\}}} \\
& 2^{\left(f\left(\boldsymbol{\zeta}_{\mp \epsilon_{0}}\right)-f\left(\boldsymbol{\zeta}_{\epsilon_{1}}\right)+\epsilon_{2}\right) R} \frac{\operatorname{Vol}\left\{\mathcal{O}_{\tau}\right\}}{\operatorname{Vol}\left\{\mathcal{O}_{\tau, \epsilon_{1}} \cap I_{\epsilon_{2}}\right\}} .
\end{aligned}
$$

Note that the three terms on the right hand sides of (77) decay exponentially with $2^{\epsilon_{0} R}$ for a given $\epsilon_{0}$. We obtain that

$$
\limsup _{\substack{\rho \rightarrow \infty \\(R, \rho) \in \mathcal{R}(k)}} \frac{\log \left(1+\frac{O_{-}(R, \rho)}{O_{+}(R, \rho)}+\frac{O_{ \pm}(R, \rho)}{O_{+}(R, \rho)}+\frac{O_{\mp}(R, \rho)}{O_{+}(R, \rho)}\right)}{\log \rho}=0 .
$$

Now, we focus on the supremum of $\frac{\log O_{+}(R, \rho)}{\log \rho}$. In region $\mathcal{O}_{\tau, \epsilon_{0}}$, there exists

$$
O_{+}(R, \rho) \leq\left(\frac{K_{\tau}}{\rho}\right)^{N+1} 2^{\left(f\left(\boldsymbol{\zeta}_{\epsilon_{0}}\right)-c(k)\right) R} .
$$

We have

$$
\begin{aligned}
& \frac{\log P_{o}(R, \rho)-c(k) R}{\log \rho} \leq \\
& \quad \frac{\log K_{\tau}^{N+1}}{\log \rho}-(N+1)+\left(f\left(\boldsymbol{\zeta}_{\epsilon_{0}}\right)-c(k)\right) \frac{R}{\log \rho} .
\end{aligned}
$$

Thus, we obtain

$$
\begin{aligned}
\limsup _{\substack{\rho \rightarrow \infty \\
(R, \rho) \in \mathcal{R}(N>k \geq 0)}} \frac{\log P_{o}(R, \rho)-c(k) R}{\log \rho} \leq \\
-(N+1)+\left(1+\frac{l N}{l-1}-c(k)\right) \times \limsup _{\substack{\rho \rightarrow \infty \\
(R, \rho) \in \mathcal{R}(N>k \geq 0)}} \frac{R}{\log \rho}, \\
\limsup _{\substack{\rho \rightarrow \infty \\
(R, \rho) \in \mathcal{R}(k=N)}} \frac{\log P_{o}(R, \rho)-c(k) R}{\log \rho} \leq \\
-1+(1-c(k)) \times \underset{\substack{\rho \rightarrow \infty \\
(R, \rho) \in \mathcal{R}(k=N)}}{\limsup } \frac{R}{\log \rho} .
\end{aligned}
$$

In summary, (63), (64), (81), and (82) reveal that the operating regions in SAF protocol have the forms (9), while $c(k)$ and $g(k)$ have the values shown in (10), and we get the proof. 


$$
\begin{aligned}
& \mathcal{O}_{\tau,-\epsilon_{0}} \triangleq\left\{(\bar{\beta}, \boldsymbol{\alpha}, \boldsymbol{\beta}) \in \mathcal{O}_{\tau} \mid \exists \epsilon_{0}>0 \text {, s.t. } \frac{\log \rho}{R}+\epsilon_{0}<\bar{\beta}, \frac{\log \sqrt{\tau} \rho}{R}+\frac{\epsilon_{0}}{2}<\max \left\{\alpha_{\max }, \beta_{\max }\right\}\right\}, \\
& \mathcal{O}_{\tau, \pm \epsilon_{0}} \triangleq\left\{(\bar{\beta}, \boldsymbol{\alpha}, \boldsymbol{\beta}) \in \mathcal{O}_{\tau} \mid \exists \epsilon_{0}>0 \text {, s.t. } \frac{\log \rho}{R}+\epsilon_{0}<\bar{\beta}, \frac{\log \sqrt{\tau} \rho}{R}+\frac{\epsilon_{0}}{2} \geq \max \left\{\alpha_{\max }, \beta_{\max }\right\}\right\}, \\
& \mathcal{O}_{\tau, \mp \epsilon_{0}} \triangleq\left\{(\bar{\beta}, \boldsymbol{\alpha}, \boldsymbol{\beta}) \in \mathcal{O}_{\tau} \mid \exists \epsilon_{0}>0 \text {, s.t. } \frac{\log \rho}{R}+\epsilon_{0} \geq \bar{\beta}, \frac{\log \sqrt{\tau} \rho}{R}+\frac{\epsilon_{0}}{2}<\max \left\{\alpha_{\max }, \beta_{\max }\right\}\right\}, \\
& \mathcal{O}_{\tau, \epsilon_{0}} \triangleq\left\{(\bar{\beta}, \boldsymbol{\alpha}, \boldsymbol{\beta}) \in \mathcal{O}_{\tau} \mid \exists \epsilon_{0}>0 \text {, s.t. } \frac{\log \rho}{R}+\epsilon_{0} \geq \bar{\beta}, \frac{\log \sqrt{\tau} \rho}{R}+\frac{\epsilon_{0}}{2} \geq \max \left\{\alpha_{\max }, \beta_{\max }\right\}\right\} \\
& \mathcal{R}_{1, \delta}(k) \triangleq \begin{cases}\left\{(R, \rho) \mid \frac{1}{\delta}>\frac{\log \rho}{R}>\frac{l N}{l-1+N-m}+\delta\right\}, & k=0, \\
\left.(R, \rho) \mid \frac{l N}{(l-1+N-m) k}-\delta>\frac{\log \rho}{R}>\frac{l N}{(l-1+N-m)(k+1)}+\delta\right\}, & 0<k<m, \\
\left.(R, \rho) \mid \frac{l N}{(l-1-m) k+m N}-\delta>\frac{\log \rho}{R}>\frac{l N}{(l-1-m)(k+1)+m N}+\delta\right\}, & m \leq k<N, \\
\left.(R, \rho) \mid \frac{l}{l-1}-\delta>\frac{\log \rho}{R}>1+\delta\right\}, & k=N, \\
\left.(R, \rho) \mid 1-\delta>\frac{\log \rho}{R}>\delta\right\}, & \text { others }\end{cases}
\end{aligned}
$$

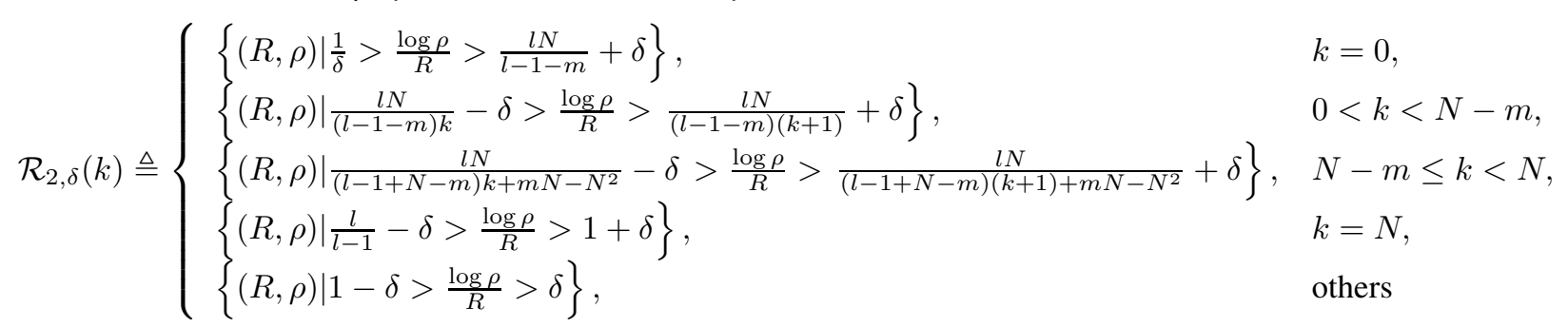

\section{APPENDIX C}

PROOF OF THEOREM 2

Similar to (58) in Appendix B, we can derive two expressions of $\mathcal{R}_{\delta}(k)$, i.e., $\mathcal{R}_{1, \delta}(k)$ and $\mathcal{R}_{2, \delta}(k)$ for the two schemes, respectively, which are shown by (83) and (84).

Let $\delta$ go to zero, and we get the operating regions $\mathcal{R}_{1}$ and $\mathcal{R}_{2}$ in Theorem 2. Next, we determine the function $f\left(\boldsymbol{\zeta}_{\epsilon_{0}}\right)$ in different operating regions. (Note that the function $f(\cdot)$ and $\boldsymbol{\zeta}_{\epsilon_{0}}$ are defined in (50) and (55), respectively.) We determine $f\left(\boldsymbol{\zeta}_{\epsilon_{0}}\right)$ by following the similar steps of the proof of Theorem 1. Then we obtain $c(k)$ and $g(k)$ based on $f\left(\boldsymbol{\zeta}_{\epsilon_{0}}\right)$ in each operating region.

\section{APPENDIX D}

\section{PROOF OF THEOREM 3}

We rewrite $\mathcal{R}_{1, \delta}(k)$ and $\mathcal{R}_{2, \delta}(k)$ in (83) and (84) by replacing $m$ with $l-1$. We fist focus on $\mathcal{R}_{1, \delta}(k)$. We can see that the operating regions indexed by $k(m \leq k<N)$ in $\mathcal{R}_{1, \delta}(k)$ are disappeared (actually these regions are degraded to a point where $\frac{\log \rho}{R}=\frac{l}{l-1}$ ). After these operating regions being removed, there are $l$ non-trivial operating regions as

$$
\begin{aligned}
& \mathcal{R}_{1, \delta}(k) \triangleq \\
& \begin{cases}\left\{(R, \rho) \mid \frac{1}{\delta}>\frac{\log \rho}{R}>l+\delta\right\}, & k=0, \\
\left.(R, \rho) \mid \frac{l}{k}-\delta>\frac{\log \rho}{R}>\frac{l}{k+1}+\delta\right\}, & 0<k<l-1, \\
\left.(R, \rho) \mid \frac{l}{l-1}-\delta>\frac{\log \rho}{R}>1+\delta\right\}, & k=l-1, \\
\left.(R, \rho) \mid 1-\delta>\frac{\log \rho}{R}>\delta\right\}, & \text { others. }\end{cases}
\end{aligned}
$$

Then we focus on $\mathcal{R}_{2, \delta}(k)$. We can see the operating regions indexed by $k(0 \leq k<N-m)$ dose not exist since in these regions $\frac{\log \rho}{R}=\infty$. Then operating regions actually begin from $k=N-m$. Therefore, we have $\mathcal{R}_{2, \delta}(k)$ as shown in (86). We can see there are $l$ non-trivial operating regions in $\mathcal{R}_{2, \delta}(k)$. We further replace $k$ with $k+(N-m)$ and then we have

$$
\begin{aligned}
& \mathcal{R}_{2, \delta}(k) \triangleq \\
& \begin{cases}\left\{(R, \rho) \mid \frac{1}{\delta}>\frac{\log \rho}{R}>\frac{l}{k+1}+\delta\right\}, & 0 \leq k<l-1, \\
\left\{(R, \rho) \mid \frac{l}{l-1}-\delta>\frac{\log \rho}{R}>1+\delta\right\}, & k=l-1, \\
\left\{(R, \rho) \mid 1-\delta>\frac{\log \rho}{R}>\delta\right\}, & \text { others. }\end{cases}
\end{aligned}
$$

By comparing (85) with (87), we can see that the BCU and WCU have the same divisions of operating regions. Let $\delta$ go to zero, and we get the operating regions $\mathcal{R}_{1}$ and $\mathcal{R}_{2}$ in Theorem 3. We can further determine $c(k)$ and $g(k)$ in each operating region by replacing $m$ with $l-1$.

\section{APPENDIX E PROOF OF THEOREM 4}

Due to the page limit, we only give a brief proof the Theorem. Based on the received signals, we can obtain the lower bound and the upper bound of the outage probability by following the same method as Appendix B. The operating 


$$
\mathcal{R}_{2, \delta}(k) \triangleq \begin{cases}\left\{(R, \rho) \mid \frac{l N}{(l-1+N-m) k+m N-N^{2}}-\delta>\frac{\log \rho}{R}>\frac{l N}{(l-1+N-m)(k+1)+m N-N^{2}}+\delta\right\}, & N-m \leq k<N, \\ \left.(R, \rho) \mid \frac{l}{l-1}-\delta>\frac{\log \rho}{R}>1+\delta\right\}, & k=N, \\ \left.(R, \rho) \mid 1-\delta>\frac{\log \rho}{R}>\delta\right\}, & \text { others }\end{cases}
$$

region $\mathcal{R}_{\delta}(k)$ with $k \in\{0, \cdots, N\}$ is defined as

$$
\begin{aligned}
& \mathcal{R}_{\delta}(k) \triangleq \\
& \begin{cases}\left\{(R, \rho) \mid \frac{1}{\delta}>\frac{\log \rho}{R}>2 N+\delta\right\}, & k=0, \\
\left.(R, \rho) \mid \frac{2 N}{k}-\delta>\frac{\log \rho}{R}>\frac{2 N}{k+1}+\delta\right\}, & 0<k<N, \\
\left.(R, \rho) \mid 2-\delta>\frac{\log \rho}{R}>1+\delta\right\}, & k=N, \\
\left.(R, \rho) \mid 1-\delta>\frac{\log \rho}{R}>\delta\right\}, & \text { others, }\end{cases}
\end{aligned}
$$

where $\delta$ is an arbitrary small value. Compare (88) with (58), we can find the expressions of $c(k)$ and $g(k)$ in different operating regions $k$ and thus obtain the TRT of the NAF protocol. Then we complete the proof.

\section{REFERENCES}

[1] L. Zheng and D. Tse, "Diversity and multiplexing: a fundamental tradeoff in multiple-antenna channels," IEEE Trans. Inf. Theory, vol. 49, no. 5, pp. 1073-1096, May 2003.

[2] J. Laneman, D. Tse, and G. Wornell, "Cooperative diversity in wireless networks: efficient protocols and outage behavior," IEEE Trans. Inf. Theory, vol. 50, no. 12, pp. 3062-3080, Dec. 2004.

[3] M. Yuksel and E. Erkip, "Multiple-antenna cooperative wireless systems: a diversity-multiplexing tradeoff perspective," IEEE Trans. Inf. Theory, vol. 53, no. 10, pp. 3371-3393, Oct. 2007.

[4] K. Azarian, H. El Gamal, and P. Schniter, "On the achievable diversitymultiplexing tradeoff in half-duplex cooperative channels," IEEE Trans. Inf. Theory, vol. 51, no. 12, pp. 4152-4172, Dec. 2005.

[5] D. Chen, K. Azarian, and J. Laneman, "A case for amplify-forward relaying in the block-fading multiple-access channel," IEEE Trans. Inf. Theory, vol. 54, no. 8, pp. 3728-3733, Aug. 2008.

[6] K. Azarian, H. El Gamal, and P. Schniter, "On the optimality of the ARQ-DDF protocol," IEEE Trans. Inf. Theory, vol. 54, no. 4, pp. 17181724, Apr. 2008.

[7] O. Leveque, C. Vignat, and M. Yukksel, "Diversity-multiplexing tradeoff for the MIMO static half-duplex relay," IEEE Trans. Inf. Theory, vol. 56, no. 7, pp. 3356-3368, July 2010.

[8] S. Gharan, A. Bayesteh, and A. Khandani, "On the diversitymultiplexing tradeoff in multiple-relay network," IEEE Trans. Inf. Theory, vol. 55, no. 12, pp. 5423-5444, Dec. 2009.

[9] Q. Li, K. Li, and K. Teh, "Achieving optimal diversity-multiplexing tradeoff for full-duplex MIMO multihop relay networks," IEEE Trans. Inf. Theory, vol. 57, no. 1, pp. 303-316, Jan. 2011.

[10] D. Gunduz, M. Khojastepour, A. Goldsmith, and H. Poor, "Multi-hop MIMO relay networks: diversity-multiplexing trade-off analysis," IEEE Trans. Wireless Commun., vol. 9, no. 5, pp. 1738-1747, May 2010.

[11] S. Loyka and G. Levin, "Diversity-multiplexing tradeoff in MIMO relay channels for a broad class of fading distributions," IEEE Commun. Lett., vol. 14, no. 4, pp. 327-329, Apr. 2010.

[12] T. Kim and M. Skoglund, "On the DMT-optimality of nondynamic DF relaying in asymmetric Nakagami fading channels," IEEE Trans. Inf. Theory, vol. 56, no. 7, pp. 3304-3309, July 2010.

[13] H. Wicaksana, S. Ting, M. Motani, and Y. Guan, "On the diversitymultiplexing tradeoff of amplify-and-forward half-duplex relaying," IEEE Trans. Commun., vol. 58, no. 12, pp. 3621-3630, Dec. 2010.

[14] K. Azarian and H. El Gamal, "The throughput-reliability tradeoff in block-fading MIMO channels," IEEE Trans. Inf. Theory, vol. 53, no. 2, pp. 488-501, Feb. 2007.

[15] K. Azarian, "Outage-limited cooperative channels: Protocols and analysis," Ph.D. dissertation, Ohio State University, Columbus, OH, 2006.
[16] S. Yang and J.-C. Belfiore, "Towards the optimal amplify-and-forward cooperative diversity scheme," IEEE Trans. Inf. Theory, vol. 53, no. 9, pp. 3114-3126, Sep. 2007.

[17] _ "Optimal space-time codes for the MIMO amplify-and-forward cooperative channel," IEEE Trans. Inf. Theory, vol. 53, no. 2, pp. 647663, Feb. 2007.

[18] R. Nabar, H. Bolcskei, and F. Kneubuhler, "Fading relay channels: performance limits and space-time signal design," IEEE J. Sel. Areas Commun., vol. 22, no. 6, pp. 1099-1109, Aug. 2004.

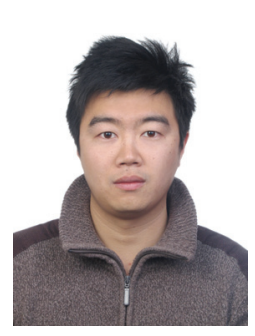

Jun Li (M’09) received his Ph.D. degree in electronic engineering from Shanghai Jiaotong University, Shanghai, P. R. China, in 2009. From January 2009 to June 2009, he worked in the Department of Research and Innovation, Alcatel Lucent Shanghai Bell, as a Research Scientist. From June 2009 to April 2012, he was a Research Postdoctoral Fellow at the School of Electrical Engineering and Telecommunications, the University of New South Wales, Australia. From April 2012 to the present, he has been a Research Associate at the School of Electrical Engineering, the University of Sydney, Australia. He has served as a Technical Program Committee member for ISCSCT2008, ICCSC 2008, APCC2009, APCC2010, VTC2011 (Spring), ICC2011, and TENCON2012. His research interests include network information theory, channel coding theory, wireless network coding, and cooperative communications.

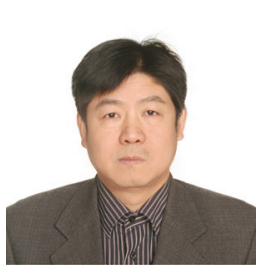

Wen Chen (M'03-SM'11) received his B.S. and M.S. from Wuhan University, China, in 1990 and 1993, respectively, and his Ph.D. from the University of Electro-Communications, Tokyo, Japan, in 1999. He was a researcher for the Japan Society for the Promotion of Sciences (JSPS) from 1999 through 2001. In 2001, he joined the University of Alberta, Canada, starting as a post-doctoral fellow in the Information Research Laboratory and continuing as a research associate in the Department of Electrical and Computer Engineering. Since 2006, he has been a full professor in the Department of Electronic Engineering, Shanghai Jiaotong University, China, where he is also the director of the Institute for Signal Processing and Systems. Dr. Chen was awarded the Ariyama Memorial Research Prize in 1997 and the PIMS Post-Doctoral Fellowship in 2001. He received the honor of "New Century Excellent Scholar in China" in 2006 and "Pujiang Excellent Scholar in Shanghai" in 2007. He was elected vice general secretary of the Shanghai Institute of Electronics in 2008. He is on the editorial board of the International Journal of Wireless Communications and Networking, and serves the Journal of Communications, Journal of Computers, Journal of Networks and EURASIP Journal on Wireless Communications and Networking as a (lead) guest editor. He is the Technical Program Committee chair for IEEE-ICCSC2008, and the General Conference Chair for IEEE-ICIS2009 and IEEE-WCNIS2010. He has published more than 100 papers in IEEE journals and conferences. His interests cover network coding, cooperative communications, cognitive radio, and MIMO-OFDM systems. 


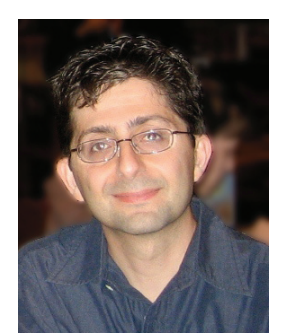

Aria Nosratinia (S'87-M'97-SM'04-F'10) is the Jonsson Distinguished Professor and associate head of the Electrical Engineering Department at the University of Texas at Dallas. He received his $\mathrm{Ph} . \mathrm{D}$. in electrical and computer engineering from the University of Illinois at Urbana-Champaign in 1996. He has held visiting appointments at Princeton University, Rice University, and UCLA. His interests lie in the broad area of information theory and signal processing, with applications in wireless communications. He was the secretary of the IEEE Information Theory Society from 2010-2011 and the treasurer for ISIT 2010 in Austin, Texas. He has served as an editor for the IEEE TRANSACTIONS ON INFORMATION THEORY and the IEEE TRANSACTIONS ON WIRELESS COMMUNICATIONS, has received a NSF CAREER award, and is a fellow of IEEE.

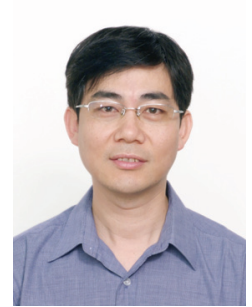

Jinhong Yuan received the B.E. and Ph.D. degrees in electronics engineering from the Beijing Institute of Technology, Beijing, China, in 1991 and 1997, respectively. From 1997 to 1999 , he was a Research Fellow at the School of Electrical Engineering, the University of Sydney, Sydney, Australia. In 2000, he joined the School of Electrical Engineering and Telecommunications, the University of New South Wales, Sydney, Australia, where he is currently a Professor for Telecommunications of the school. He has published two books, two book chapters, over 200 papers in telecommunications journals and conference proceedings, and 40 industrial reports. He is a co-inventor of one patent on MIMO systems and two patents on low-density-parity-check (LDPC) codes. He has coauthored three Best Paper Awards and one Best Poster Award, including a Best Paper Award at the IEEE Wireless Communications and Networking Conference (WCNC), Cancun, Mexico in 2011, and a Best Paper Award of the IEEE International Symposium on Wireless Communications Systems (ISWCS), Trondheim, Norway, in 2007. His publications are available from http://www2.ee.unsw.edu.au/wcl/JYuan.html. He also serves as the IEEE NSW Chair of the Joint Communications/Signal Processions/Ocean Engineering Chapter. His current research interests include error control coding and information theory, communication theory, and wireless communications. 